\title{
Sebastian Bücking* Zur Komposition von insofern als im Deutschen
}

https://doi.org/10.1515/zfs-2018-0001

\begin{abstract}
This paper is about the compositional interpretation of adverbial clauses as introduced by insofern als (lit. 'insofar as') in German (= IAs); for example, Die Lösung ist insofern attraktiv, als sie günstig ist. (lit. 'The solution is insofar attractive as it is cheap.'). I argue that IAs always contribute dimensional restrictions, but do so in two distinct structure-sensitive ways: IAs can be structurally integrated into their host clauses and, thus, dimensionally specify a lexical component of the matrix VP (= LIAs) ('The solution is attractive in price.'); alternatively, they can be structurally non-integrated and, thus, provide a dimension against which the matrix sentence is evaluated holistically (= SIAs) ('In light of the fact that the solution is cheap, one may say that it is attractive.'). The proposal is supported by both syntactic and semantic-pragmatic evidence: (i) LIAs and SIAs are distinguished by independently motivated tests for structural (non)integration such as scope of negation and particles, focusbackground structure, and variable binding. (ii) LIAs are sensitive to a lexically given multidimensional matrix predication. SIAs, by contrast, require that the embedded clause specify an objective justification for the truth of the matrix proposition as a whole. In particular, IAs are shown to not pass tests for subjectivity as discussed for finden ('find'); for example, judge-dependency, resistance against denial, or, accessibility of a subjective attitude. The paper concludes by sketching a formal derivation of both readings. Accordingly, LIAs constrain the dimensional parameter of multidimensional lexical predications, while SIAs provide facts that de re justify the assertibility of predications for topic situations.
\end{abstract}

Keywords: explicative-restricting adverbial clauses, clausal linkage, syntaxsemantics interface, (multi)dimensional predication, situation semantics

\footnotetext{
*Korrespondenzautor: Sebastian Bücking, Sonderforschungsbereich 833 „Bedeutungskonstitution“, Nauklerstraße 35, Universität Tübingen, Tübingen, Germany, E-mail: sebastian.buecking@uni-tuebingen.de
}

Ә Open Access. (c) 2018 Sebastian Bücking, publiziert von De Gruyter. (cc)BY-NC-ND Dieses Werk ist lizensiert unter der Creative Commons Attribution-NonCommercial-NoDerivatives 4.0 Lizenz. 


\section{Worum geht's?}

Gegenstand des vorliegenden Beitrags sind Gefügestrukturen mit einem insofern einschließenden Matrixsatz und einem als-eingeleiteten subordinierten Satz, s. (1) (= IA-Gefüge).

(1) Dies ist insofern erstaunlich, als die Frauen nicht gerade auf der Welle des Erfolgs schwimmen.

(Zeit, 29.3. 1985, 1; zit. nach Zifonun et al. 1997: 2327)

In der Literatur fehlt eine ausführliche Analyse der Bedeutung von IA-Gefügen; eine kompositional explizite Herleitung ihrer Interpretation an der Syntax-Semantik-Schnittstelle wurde meines Wissens bisher überhaupt noch nicht unternommen. Ziel ist es hier, zur Schließung dieser bemerkenswerten Forschungslücke beizutragen.

Einen ersten Anknüpfungspunkt liefern Zifonun et al. (1997: 2327). Sie geben für insofern als an, dass „mit dem Untersatz [...] diejenige Hinsicht genannt [wird], unter der überhaupt ein Geltungsanspruch für die Aussage erhoben werden kann“. Ähnlich weist auch die Duden-Grammatik (Duden 2016: §§946, 1804) insofern (als) eine sowohl explikative als auch restringierende Funktion zu. Diese Annäherung ist plausibel; sie wirft allerdings die Frage auf, was hier unter einer Hinsicht sowie der damit einhergehenden Beschränkung eines Geltungsanspruchs zu verstehen ist. Sicherlich zu einfach wäre die Annahme, dass der als-Satz lediglich eine Bedingung liefert, unter der die Matrixaussage wahr ist. Gegen eine solche Analyse als hypothetisches Konditional spricht zweierlei: Die als-Satz-Proposition entspricht keiner Bedingung mit offenem Wahrheitswert, sondern sie wird als wahr präsentiert. So assertiert der Sprecher mit (1), dass die Frauen nicht gerade auf der Welle des Erfolgs schwimmen. Darüber hinaus geht es in IA-Gefügen nicht einfach um die Wahrheit der Matrixaussage; vielmehr liefert der als-Satz eine Art Begründung dafür, warum man die auf der Matrixebene gewählten Worte überhaupt verwenden kann; s. dazu die präzisierte Paraphrase in (2).

(2) In Hinsicht darauf, dass die Frauen nicht gerade auf der Welle des Erfolgs schwimmen, kann man sagen, dass dies erstaunlich ist.

Diese intuitive Einschätzung schließt unmittelbar an einen zweiten Anknüpfungspunkt für die Analyse von IA-Gefügen an: Reis (2013a: 424, Anm. 37) vermerkt, dass das Fragewort inwiefern nicht auf die „Begründung von Sachverhalten, sondern [die] Begründung von Interpretationen von Sachverhalten“ ziele. 
Unterstützt wird diese Annahme von der Beobachtung, dass inwiefern mit Sachverhaltsbeschreibungen ohne Interpretationsoffenheit inkompatibel ist, s. ihr Beispiel in (3a). Offenbar analog verhält sich das hier interessierende KorrelatPendant in $(3 \mathrm{~b})$.

(3) a. \# Inwiefern ist er 1,90 m groß?

(Reis 2013a: 424, Anm. 37)

b. \# Ben ist insofern $1,90 \mathrm{~m}$ groß, als er ....

Reis sieht hierin eine mögliche Parallele zur Komplementation bei finden, dem eigentlichen Gegenstand ihrer Untersuchung. Gemäß ihrer Analyse sorgt finden für die subjektive Auswahl von Interpretationen für kontextuell gegebene Sachverhalte und ist deshalb ebenfalls nur bei interpretationsoffenen Sachverhalten lizenziert, s. (4a) vs. (4b).

(4) a. Ich finde dies erstaunlich.

b. \# Ich finde Ben 1,90 m groß.

Präzisiert werden soll im vorliegenden Beitrag zum einen, wie sich der hinsichtsbasierte Vorschlag aus Zifonun et al. (1997) zum (Reis'schen) Begriff von Interpretation verhält; dies wird ebenfalls die Frage einschließen, ob, und wenn ja, in welcher Weise mit IA-Gefügen ein metasprachlicher Effekt verknüpft ist. Zum anderen muss überprüft werden, wie weit die (suggerierte) Parallele zwischen IA-Gefügen und finden bezüglich Subjektivität tatsächlich trägt. Beachtenswert ist gleich auf den ersten Blick, dass IA-Gefüge anders als finden keinen Einstellungsträger explizit machen.

Quer zu den bisherigen Überlegungen liegt eine meines Wissens in der Literatur bisher nicht registrierte weitere Beobachtung. ${ }^{1}$ Für (1) ist es plausibel, dass der als-Satz einen holistischen Bezug zur Aussage im Matrixsatz hat; wie von der Paraphrase in (2) nahegelegt, begründet also der als-Satz die Behauptbarkeit der Aussage, dass etwas erstaunlich ist, als Ganze. Anders in (5a): Hier wird auf der Basis des als-Satzes das eingebettete Prädikat selbst beschränkt; mit anderen Worten: Das IA-Gefüge sorgt dafür, dass eine dimensional spezifizierte Interpretation des lexikalischen Prädikats - es geht um zeitliche, nicht

1 Verwiesen sei lediglich auf Bartsch (1986), die im Ansatz ähnliche Überlegungen zu dimensionalen Restriktoren wie with regard to health oder healthwise anstellt. Das ist aber m.E. kaum ausgearbeitet, s. dazu auch Abschnitt 4.3 und Fußnote 19. 
aber preisliche Attraktivität - herausgegriffen und über den Subjektreferenten prädiziert wird, s. die Paraphrase in (5b).

(5) a. Die Lösung ist insofern attraktiv, als sie schnell umsetzbar ist. Sie ist hingegen insofern unattraktiv, als sie teuer ist.

b. Die Lösung ist in einer Hinsicht attraktiv, die sich daraus ergibt, dass sie schnell umsetzbar ist. Sie ist hingegen in einer Hinsicht unattraktiv, die sich aus ihrem hohen Preis ergibt.

Dies legt die Unterscheidung von sententialen insofern-als-Gefügen (= SIA-Gefügen) und insofern-als-Gefügen mit lexikalischem Bezug (= LIA-Gefügen) nahe. Dass es sich dabei um eine sprachlich relevante Unterscheidung handelt, sei bereits hier an zwei weiteren Beobachtungen illustriert. Aufschlussreich ist zum einen der Blick auf lexikalische Prädikate wie schwer, die intuitiv keine dimensionale Spezifizierung erlauben; entsprechend können Beispiele wie in (6) nur holistisch, d.h. als SIA-Gefüge interpretiert werden.

(6) Ben ist insofern schwer, als er hundert Kilo wiegt.

$\neq$,Ben ist in einer Hinsicht schwer, die sich daraus ergibt, dass er hundert Kilo wiegt.‘

$=$,In Hinsicht darauf, dass Ben hundert Kilo wiegt, kann man sagen, dass Ben schwer ist.'

Zum anderen ist bemerkenswert, dass sich die beiden angedeuteten Lesarten auch kombinieren lassen, s. dazu (7).

(7) Ich habe den Film nicht gesehen; ich weiß nur, er ist von Degeto produziert. Insofern ist er möglicherweise insofern langweilig, als er einen sehr konventionellen Plot hat.

Vermittelt über ein anaphorisches insofern (dazu unten mehr) wird die Tatsache, dass der Film von Degeto produziert ist, als Grund dafür angeführt, warum man sagen kann, dass der Film möglicherweise in einer bestimmten Hinsicht langweilig ist. Die holistische SIA-Interpretation wird dadurch unterstrichen, dass der Möglichkeitsoperator hier im Skopus von insofern steht. Hingegen ist das zweite Vorkommen von insofern - das klar Skopus unterhalb von möglicherweise hat - an die Auswahl einer spezifischen Langeweile-Hinsicht gekoppelt; es liegt also eine LIA-Interpretation vor. Der enge Skopus unterhalb von möglicherweise zeigt zudem, dass bei LIA-Interpretation die als-Satz-Proposition nicht mehr als wahr assertiert wird. 
Eine wesentliche Anschlussfrage lautet, ob die Unterscheidung von SIAund LIA-Interpretationen kompositional motiviert ist. Es liegt nahe, die Interpretation mit lexikalischem Bezug an den strukturell ermöglichten Zugriff des insofern als-Satzes auf den deskriptiven lexikalischen Kern des Matrixsatzes zurückzuführen. Dies setzt Integration des insofern als-Satzes in seinen Bezugssatz voraus; erste gute Evidenz dafür liefern der enge Skopus relativ zu möglicherweise sowie die fehlende eigene Sprechaktgeltung. Demgegenüber sollte die sententiale Interpretation mit einer Gefügestruktur korrelieren, gemäß der der insofern als-Satz syntaktisch auf den Matrixsatz als Ganzen zielt. Dies ist mit Desintegration des insofern als-Satzes kompatibel; der relativ zu möglicherweise weite Skopus sowie die eigene Sprechaktgeltung stützen diese Annahme. Mit diesen Überlegungen schließt der vorliegende Beitrag an prominente Debatten zu Satzfügungseigenschaften und ihren möglichen Interpretationseffekten an. Das vielleicht bekannteste Beispiel liefern weil-Sätze, für die der Zusammenhang zwischen Fügungseigenschaften und Lesartenvarianz nach wie vor kontrovers diskutiert wird (s. neben vielen anderen Antomo und Steinbach [2010], Reis [2013b]). Eine direkte Parallele zur hier relevanten Gegenüberstellung zwischen lexikalischem und sententialem Bezug liefern hypothetische Vergleichssätze, s. (8). Bei Integration wie in (8a) (markiert durch prosodische Integration) wird der Vergleichssatz als modale Adverbiale und damit als Modifikator zum Verb interpretiert; bei Desintegration wie in (8b) (markiert durch prosodische Eigenständigkeit) liegt hingegen Bezug auf die Aussage als Ganze vor; s. zur ausführlichen Diskussion dieser syntaktischen Differenzierung und ihrer semantischen Konsequenzen Demske (2014), Bücking (2015, 2017).

(8) a. Ben fährt Rad, als sei er beTRUNken.

,Ben fährt auf eine Art und Weise Rad, wie er Rad fahren würde, wenn er betrunken ist.'

(Bücking 2017: [1c]/[3])

b. Ben SCHWEIGT, als sei er beLEIdigt.

,Ben schweigt; eine Situation dieser Art läge auch vor, wenn er beleidigt ist.

(Bücking 2017: [32]/[36b])

Die zentrale Herausforderung besteht also darin, die unterstellte Korrelation zwischen Gefügestruktur und Lesarten für IA-Gefüge zu verifizieren und die jeweils relevante Interpretation auf der Basis eines gemeinsamen invarianten Bedeutungskerns für insofern als systematisch abzuleiten.

Zwei Aspekte der Analyse von IA-Gefügen werde ich im Folgenden nicht diskutieren: Außen vor bleibt die Frage, wie sich IA-Gefüge zu ihren auf den 
ersten Blick (nahezu) bedeutungsgleichen Konstruktionsvarianten mit dass bzw. insoweit verhalten, s. (9). Zu klären ist hier, ob es sich dabei um bloß stilistische Alternativen handelt oder mit der Wahl von als vs. (als) dass bzw. insofern vs. insoweit feinkörnige Bedeutungsunterschiede transportiert werden können.

(9) a. Die Lösung ist insofern attraktiv, \{dass / als dass $\}$ sie schnell umsetzbar ist.

b. Die Lösung ist insoweit attraktiv, als sie schnell umsetzbar ist.

Unberücksichtigt bleiben außerdem konditionale Gefüge mit stets endbetonter Subjunktion soFERN (seltener insoFERN), s. (10). ${ }^{2}$

(10) $\{$ SoFERN / InsoFERN\} sie schnell umsetzbar ist, ist die Lösung attraktiv.

Diese sind anders als IA-Gefüge hypothetisch. Offen bleibt damit vor allem die Frage, ob sich vielleicht dennoch ein systematischer Zusammenhang zwischen IA-Gefügen und ihrem hypothetischen Pendant herstellen lässt. ${ }^{3}$

Zum Argumentationsgang im Einzelnen: In Abschnitt 2 möchte ich die Distinktion zwischen LIA- und SIA-Gefügen über strukturbezogene Indizien unabhängig motivieren und dabei zeigen, dass LIA-Sätze integrierte und SIA-Sätze desintegrierte Adjunkte sind. In Abschnitt 3 wird die Sensitivität von IA-Gefügen bezüglich verschiedener Hinsichten präzisiert. Dazu soll - auch relativ zum Verhalten von finden - zweierlei geklärt werden: Welche Optionen und Beschränkungen regulieren die Kombinatorik mit lexikalisch angelegter (Mehr)Dimensionalität (Abschnitt 3.1)? Welches Verhältnis besteht zwischen dimensionaler Spezifizierung und (subjektiver) Auswahl von Interpretationen (Abschnitt 3.2)? Ich werde dafür argumentieren, dass LIA-Sätze mehrdimensionale lexikalische Prädikationen dimensional beschränken, SIA-Sätze hingegen sentential gegebene Sachverhaltsbeschreibungen dimensional beschränken. Dabei hat die jeweilige Dimensionseinschränkung den metasprachlichen Effekt, dass der

2 Das Duden-Aussprachewörterbuch sieht wie in (10) für das subjunktionale insofern Endbetonung vor, erlaubt allerdings als Alternative ebenfalls initialen oder medialen Akzent, s. Duden (2015: 471). Demgegenüber wird für das hier im Vordergrund stehende korrelierende insofern als primäre Variante die mediale Betonung auf so angegeben, während Initial- und Endakzent als Alternativen genannt werden. Im vorliegenden Artikel folge ich der primär angegebenen Standardlautung, die eine phonologische Differenzierung zwischen Korrelat und Subjunktion vorsieht.

3 Auch zu sofern gibt es meines Wissens wenig Literatur; einen deskriptiven Überblick über Verwendungsoptionen liefern Breindl et al. (2014: Kap. C4.1). 
Sprecher Interpretationen einer lexikalischen Prädikation bzw. einer Sachverhaltsbeschreibung herausgreift. Gleichzeitig leitet sich die für SIA-Sätze charakteristische Begründungsintuition aus der konstitutiven Rolle der Dimensionseinschränkung für die ausgewählte Interpretation ab. Insbesondere wird gezeigt, dass IA-Gefüge - anders als finden - keine explizite Subjektivitätskomponente involvieren. In Abschnitt 4 wird ein formalsemantischer Vorschlag skizziert, der über einen uniformen Lexikoneintrag für insofern als die LesartenUnterschiede aus den unterschiedlichen Fügungseigenschaften ableitet. Das Fazit in Abschnitt 5 fasst die Ergebnisse zusammen.

\section{Zur unterschiedlichen Gefügestruktur von SIA- und LIA-Gefügen}

Die folgende Bestandsaufnahme zur Gefügestruktur von IA-Gefügen basiert im Wesentlichen auf etablierten (Des)Integrationstests, s. Reich und Reis (2013) zu einem Überblick. Im Vordergrund steht die Motivation für eine je nach LIAoder SIA-Interpretation verschiedene kompositionale Ausgangsposition, nicht eine vollständige syntaktische Analyse.

\subsection{Anschlussoptionen und Reformulierungen}

SIA-Gefüge lizenzieren die Reformulierung über ein anaphorisches insofern, das dem ursprünglichen Matrixsatz desintegriert vorgeschaltet ist, s. (11). Diese Reformulierung korreliert klar mit der für SIA-Gefüge angesetzten Interpretation: Es werden Gründe für die Assertierbarkeit der Matrixaussage als Ganzer geliefert.

(11) a. Ben ist gesund, hat einen tollen Job und ist frisch verliebt. Insofern: Ihm geht's gut.

,In Hinsicht darauf, dass Ben gesund ist, einen tollen Job hat und frisch verliebt ist, kann man sagen, dass es ihm gut geht.'

b. Die Lösung ist günstig und schnell umsetzbar. Insofern: Sie ist attraktiv. ,In Hinsicht darauf, dass die Lösung günstig und schnell umsetzbar ist, kann man sagen, dass sie attraktiv ist.`

Man mag einwenden, dass man für z. B. (11a) ebenfalls darauf schließen kann, dass es Ben gesundheitlich und beruflich gut geht. Der Weg zu dieser Interpretation erfolgt aber nicht über die direkte dimensionale Einschränkung des Prä- 
dikats gut, sondern auf der Basis der spezifisch begründeten Matrixaussage als Ganzer.

Demgegenüber zielt in LIA-Gefügen die Einschränkung unmittelbar auf das eingebettete lexikalische Prädikat des Matrixsatzes. In dieser Lesart lässt sich der Ausschluss alternativer Hinsichts-Interpretationen über die Fokuspartikel nur und damit Matrixsatz-intern explizieren, s. (12). Der folgende Satz kann dabei typischerweise durch ansonsten eingeleitet werden.

(12) a. Ben geht es nur insofern gut, als er gesund ist. Ansonsten geht's ihm schlecht: Seine Freundin hat ihn verlassen und seine Chefin hat ihn rausgeschmissen.

,Ben geht es nur in der Hinsicht gut, die sich daraus ergibt, dass er gesund ist.‘

b. Die Lösung ist nur insofern attraktiv, als sie günstig ist. Ansonsten ist sie aber Mist.

,Die Lösung ist nur in der Hinsicht attraktiv, die sich daraus ergibt, dass sie günstig ist.‘

Betont sei, dass die in den Beispielen verhandelten Hinsichten und Prädikationen nicht als solche zwingend auf die LIA- oder SIA-Lesart festlegen, sondern ein enormes Anpassungspotenzial besteht. Zwar unterstützt die Explizierung einer Hinsicht in (12) den lexikalischen, die Angabe mehrerer Hinsichten in (11) den holistischen Bezug, doch ist diese Korrelation keineswegs zwingend. So kann z. B. die Basis der LIA-Gefüge in (12) jeweils auch als Basis für SIA-Lesarten dienen, s. die folgenden Reformulierungen über ein desintegriertes anaphorisches insofern:

(13) a. Ben ist gesund. Insofern: Ihm geht's gut. (\#Ansonsten geht's ihm aber nicht so gut.)

b. Die Lösung ist günstig. Von dem her: Sie ist attraktiv. (\#Ansonsten ist sie aber Mist.)

Diese Flexibilität gründet darauf, dass man die hier ausgewählte Hinsicht auch als einzig relevante für die holistische Evaluation der Bezugsaussage konzipieren kann. Mit der holistischen SIA-Interpretation korreliert, dass alternative lexikalisch basierte Hinsichten implizit als irrelevant erscheinen; entsprechend ist ein anschließendes ansonsten abweichend.

Für die SIA-Interpretation ist also zentral, dass sich der Sprecher auf die Matrixproposition als Ganze verpflichtet; dies führt dazu, dass er sich nicht mehr ohne Weiteres - etwa durch ein unspezifiziertes ansonsten wie in (13) - 
von ihr distanzieren kann. Allerdings signalisiert der Sprecher durch die Relativierung auf die via als-Satz eingeführte tatsachenbasierte Hinsicht dennoch eine Einschränkung. Dies eröffnet marginal auch für SIA-Gefüge - trotz holistischer Deutung - die gleichzeitige Behauptbarkeit sich eigentlich ausschließender Matrixaussagen, s. (14):

(14) Wie geht es Ben? - Ben ist gesund. Insofern: Ihm geht's gut. Aber er hat kürzlich seinen Job verloren. Insofern: Ihm geht's nicht gut.

Der Sprecher artikuliert mit (14), dass Fakt A die Behauptbarkeit von $p$, Fakt B aber die Behauptbarkeit von $\neg p$ rechtfertigt. Er gerät so in keinen Widerspruch, sondern verweist darauf, dass man - je nach gewählter faktbasierter Perspektive - Verschiedenes behaupten kann. Der Diskurs ist dennoch relativ markiert, eben weil man dem Sprecher unterstellen muss, dass er sich zwischen den holistischen Perspektiven nicht entscheiden kann. Man beachte entsprechend, dass die SIA-Lesart nicht mit der unauffälligeren lexikalischen Einschränkung in (15) zusammenfällt:

(15) Wie geht es Ben? - Ben geht es gesundheitlich, nicht aber beruflich gut. Der Gesundheitscheck war erfolgreich; gleichzeitig hat er aber seinen Job verloren.

Hier wird via Adjektiv das Prädikat gut geh- selbst dimensional spezifiziert, sodass überhaupt kein Anspruch auf eine holistische Interpretation erhoben wird und damit von vornherein keine Gefahr eines inneren Widerspruchs besteht.

\subsection{Fokuspartikel und Negationsskopus}

Bereits die Beispiele in (12) deuten darauf, dass allein LIA-Gefüge im Skopus von Fokuspartikeln stehen können. Das Beispiel in (16) bekräftigt diese These: Die Fokuspartikel sogar erlaubt nur die LIA-, nicht aber die SIA-Paraphrase.

(16) Die Lösung ist sogar insofern attraktiv, als sie günstig ist.

$=$,Die Lösung ist sogar in einer Hinsicht attraktiv, die sich daraus ergibt, dass sie günstig ist.'

F ,Sogar in Hinsicht darauf, dass die Lösung günstig ist, kann man sagen, dass sie attraktiv ist. 
Ein ähnlich klares Bild liefert Negationsskopus. So kann Negation in LIA-Gefügen problemlos oberhalb der von insofern bestimmten Restriktion skopieren, s. (17), was gleichzeitig die Fortführung mit sondern nahelegt.

(17) a. Die Lösung ist nicht insofern attraktiv, als sie günstig ist, sondern insofern, als sie schnell umsetzbar ist.

,Die Lösung ist nicht in einer Hinsicht attraktiv, die sich aus ihrem niedrigen Preis ergibt, sondern in einer, die sich aus ihrer schnellen Umsetzbarkeit ergibt.'

b. Ben geht es nicht insofern gut, als er frisch verliebt ist, sondern insofern, als er Urlaub hat.

,Ben geht es nicht in einer Hinsicht gut, die sich aus einer frischen Verliebtheit ergibt, sondern in einer, die sich aus seinem Urlaub ergibt.

SIA-Gefüge hingegen verbieten einen weiten Negationsskopus. So kann der Sprecher mit den gegebenen Beispielen nicht zurückweisen, dass man relativ zur vom als-Satz bestimmten Tatsache die Matrixaussage als Ganze behaupten kann:

(18) a. Die Lösung ist nicht insofern attraktiv, als sie günstig ist.

₹ ,Es ist nicht so, dass man in Hinsicht darauf, dass die Lösung günstig ist, sagen kann, dass sie attraktiv ist.'

b. Ben geht es nicht insofern gut, als er frisch verliebt ist.

$\neq$,Es ist nicht so, dass man in Hinsicht darauf, dass Ben frisch verliebt ist, sagen kann, dass es ihm gut geht.

Demgegenüber erlaubt die SIA-Interpretation erwartungsgemäß ohne Weiteres engen Skopus der Negation, s. (19) (zum Verhältnis von LIA-Gefügen und engem Negationsskopus s. Abschnitt 3.1.2).

(19) a. Die Lösung ist insofern nicht unattraktiv, als sie günstig ist.

,In Hinsicht darauf, dass die Lösung günstig ist, kann man sagen, dass sie nicht unattraktiv ist.

b. Ben geht es insofern nicht gut, als ihn kürzlich seine Freundin verlassen hat.

,In Hinsicht darauf, dass ihn seine Freundin kürzlich verlassen hat, kann man sagen, dass es Ben nicht gut geht. 


\subsection{Projektion innerhalb lexikalischer Phrasenkategorien}

Die Distinktion zwischen lexikalisch zu verankernder und sententialer Restriktion sagt voraus, dass ein syntaktisch forcierter Bezug der Restriktion auf den lexikalischen Anker nur mit der LIA-Interpretation verträglich ist. AP-Topikalisierung wie in (20) liefert eine solche Konfiguration und sorgt tatsächlich eindeutig für die LIA-Lesart. Die entsprechende Topikalisierung ist allerdings informationsstrukturell zu lizenzieren. So kann entweder die Auswahl einer Hinsicht - s. (20a) mit Fokusexponent innerhalb des als-Satzes - oder die Auswahl des Prädikationstargets - s. (20b) mit Fokusexponent im Matrixsatz - zur Debatte stehen; s. Abschnitt2.4 für Details zu Fokus-Hintergrund-Gliederung und Stellungsverhalten. ${ }^{4}$

(20) a. Insofern attraktiv, als sie GÜNstig ist, ist die Bremer Lösung.

$=$,Die Bremer Lösung ist in einer Hinsicht attraktiv, die sich daraus ergibt, dass sie günstig ist.

\# ,In Hinsicht darauf, dass sie günstig ist, kann man sagen, dass die Bremer Lösung attraktiv ist.'

b. Insofern gut, als er frisch verliebt ist, geht es BEN.

$=$,Ben geht es in einer Hinsicht gut, die sich daraus ergibt, dass er frisch verliebt ist.

\# ,In Hinsicht darauf, dass er frisch verliebt ist, kann man sagen, dass es Ben gut geht.‘

Ein analoges Bild liefert die NP-interne Projektion in (21): Sie unterstützt den Skopus der Restriktion über das lexikalische Prädikat, konfligiert aber mit einem holistischem Bezug auf den Matrixsatz. Entsprechend ist allein die LIAInterpretation zugänglich.

4 Erwähnt sei bereits hier, dass LIA-Beispiele mit engem Fokus auf dem Prädikationstarget wie (20b) ein inhärentes Interpretationsproblem mit sich bringen: Der enge Fokus auf dem Subjekt setzt voraus, dass die dimensional spezifizierte Prädikation gegeben ist und zur Debatte steht, welches Subjekt sie erfüllt. Gleichzeitig aber prädiziert der als-Satz spezifische Informationen über das jeweilige Subjekt selbst, was ein Gegebensein der Prädikation über Subjekte hinweg eigentlich unmöglich macht. Dieser Punkt wird dann klar, wenn man versucht, die pronominale Referenz in (20b) aufzulösen: Man muss einerseits annehmen, dass er variabel identifiziert wird, denn sonst würde die Hinsicht nicht als verallgemeinerbar taugen. Gleichzeitig muss aber das Pronomen mit dem jeweiligen Subjekt koindiziert werden; schließlich geht es Ben ja nicht in einer Hinsicht gut, die sich aus der Verliebtheit von irgendwem ergibt, sondern daraus, dass er selbst verliebt ist. Nach meiner Intuition hat man als Leser auch tatsächlich entsprechend Probleme, die pronominale Referenz in (20b) zu fixieren. 
(21) Ben ist ein insofern attraktiver Mann, als er sehr hübsch ist.

$=$,Ben ist in einer Hinsicht ein attraktiver Mann, die sich aus seinem hübschen Aussehen ergibt.

₹ ,In Hinsicht darauf, dass Ben hübsch ist, kann man sagen, dass er ein attraktiver Mann ist.

\subsection{Fokus-Hintergrund-Gliederung und Stellungsverhalten}

Ich gehe mit u. a. Reich und Reis (2013: 551) sowie Reis und Wöllstein (2010: 147-148) davon aus, dass in desintegrierten Satzgefügen der abhängige Satz sowie der Matrixsatz eigene Fokus-Hintergrund-Gliederungen bilden und deshalb separate Hauptakzente tragen müssen. Demgegenüber können integrierte abhängige Sätze prosodisch integriert werden. Die entsprechende Diagnose wird allerdings durch zwei Faktoren verkompliziert: Zum einen können sich auch integrierte Gefügestrukturen - insbesondere längere Einheiten - in separate Akzentphrasen gliedern, sodass der prosodische Unterschied zur desintegrierten Fügung verunklart wird, s. mit Literaturverweisen Reis und Wöllstein (2010: Anm. 19). Zum anderen kann in desintegrierten Gefügen semantisch lizenzierte progrediente Betonung den Eindruck vermitteln, der desintegrierte Satz sei in die Fokus-Hintergrund-Gliederung des Matrixsatzes integriert; so verweist z. B. Reis (2011: 325) für kaum-als-Gefüge darauf, dass hier semantische Anforderungen über Desintegration hinweg für progrediente Prosodie sorgen können. Wie verhalten sich hier nun IA-Gefüge?

LIA-Gefüge erlauben eine Hutkontur aus steigendem Akzent auf insofern und fallendem Akzent im als-Satz und so die Bildung nur einer Fokus-Hintergrund-Gliederung, s. (22).

(22) a. Ben geht es in/SOfern gut, als er geSUND $\backslash$ ist.

,Ben geht es in einer Hinsicht gut, die sich aus seiner Gesundheit ergibt.'

b. Die Lösung ist in/SOfern attraktiv, als sie GÜN\stig ist.

,Die Lösung ist in einer Hinsicht attraktiv, die sich aus ihrem niedrigen Preis ergibt.‘

Dies liefert ein Indiz dafür, dass die LIA-Interpretation mit integrierter Satzfügung korreliert. ${ }^{5}$

5 Zentral für die Belange hier ist die mit der Phrasierung einhergehende informationsstrukturelle Einheit. Offen bleibt so, wie sich die angenommene prosodische Markierung zur Auszeichnung von Kontrasttopiks bzw. von Rahmensetzern allgemeiner verhält. Zumindest kann man sagen, dass insofern einen zentralen Aspekt beider - nämlich die explizite Anzeige einer 
Demgegenüber haben in SIA-Gefügen Matrix- und als-Satz notwendig separate Fokus-Hintergrund-Gliederungen, s. (23). Dies unterstützt die Annahme, dass die SIA-Interpretation mit Desintegration korreliert.

(23) a. Ben geht es in/SOfern gut, als er ge/SUND ist $\backslash$.

,In Hinsicht darauf, dass Ben gesund ist, kann man sagen, dass es ihm gut geht.

b. Die Lösung ist in/SOfern attraktiv, als sie / GÜNstig ist $\$.

,In Hinsicht darauf, dass die Lösung günstig ist, kann man sagen, dass sie attraktiv ist.

Das dabei entscheidende minimale distinktive Merkmal ist, dass der Fokusexponent im als-Satz einen steigenden Akzent aufweist und damit eine Hutkontur wie in (22) - dort bleibt die im Matrixsatz aufgebaute Höhe bis zum Fall im alsSatz erhalten - verhindert. Der Unterschied fällt nur deshalb nicht markanter aus, weil auch in (23) eine progrediente Prosodie zwingend ist. Diese lässt sich aber ohne Weiteres auf die semantische Selektionsforderung von insofern zurückführen (zur entsprechenden Semantik s. Abschnitt4) und spricht damit nicht gegen die Annahme, dass eine desintegrierte Fügungsstruktur vorliegt.

Dieses Bild bestätigt sich, wenn man (vermeintliche) Vorkommen von IASätzen im Nach-, Mittel- sowie Vorfeld untersucht, bei denen die Adjazenz von insofern und als-Satz den Progredienz-Effekt neutralisiert und damit die prosodische Differenzierung erleichtert. Zunächst zum Nachfeld und den Minimalpaaren in (24) und (25): 6

(24) a. Die Lösung ist attraktiv, in/SOfern, als sie GÜN\stig ist.

b. Die Lösung ist attrakTIV\, in/SOfern, als sie /GÜNstig ist $\$.

(25) a. Ben geht es gut, in/SOfern, als er geSUND $\backslash$ ist.

b. Ben geht es GUT\, in/SOfern, als er ge/SUND ist $\backslash$.

Sub-Optimalität in Frage-Antwort-Sequenzen à la Delimitation wie definiert in Krifka und Musan (2012) - schon lexikalisch mitbringt. Das heißt aber, dass diese lexikalische Eigenschaft auch für den SIA-Fall gelten sollte; der delimitative Charakter von insofern liegt also quer zur hier interessierenden Differenzierung der Gefügestrukturen. Ebenso scheint es mir für die Argumentation nicht ausschlaggebend zu sein, ob der steigende Akzent in der angenommenen Hutkontur ein Wurzelakzent - dabei geht dem Anstieg ein pointierter Fall voraus - sein muss oder nicht.

6 In einer Vorversion des vorliegenden Artikels habe ich diese Stellungsvariante nicht beachtet. Veronika Ehrich verdanke ich den Hinweis darauf, dass diese Option den prosodischen Unterschied vergleichsweise klar hervortreten lässt. 
Die integrierende Prosodie in (24a)/(25a) mit Hauptakzent allein im insofern alsSatz korreliert mit der LIA-Interpretation, gemäß der Hinsichten der lexikalischen Prädikate attraktiv bzw. gut herausgegriffen werden; in diesem Fall wird nach wie vor Progredienz zwischen Matrixsatz und abhängigem Satz forciert. Demgegenüber korreliert ein separater Hauptakzent im Matrixsatz wie in (24b)/ (25b) mit der holistischen SIA-Interpretation; in diesem Fall wird keine Progredienz zwischen den Sätzen forciert. Entsprechend stehen finale SIA-Sätze nicht im integrierten Nachfeld, sondern in desintegrierter Schlussstellung (s. zur entsprechenden Differenzierung u. a. Reis [1997], Reis und Wöllstein [2010]).

Analoge Beobachtungen treffen auf Kontraste im Mittelfeld wie in (26) und (27) zu.

(26) a. Die Lösung ist in/SOfern, als sie GÜN\stig ist, attraktiv.

b. Die Lösung ist - in/SOfern, als sie /GÜNstig ist - attrakTIV\.

(27) a. Ben geht es in/SOfern, als er geSUND $\backslash$ ist, gut.

b. Ben geht es - in/SOfern, als er ge/SUND ist - GUT\.

Erneut sorgt die integrierende Prosodie in (26a)/(27a) für die LIA-Interpretation. Dass hier Integration des Adverbialsatzes bei Binnenstellung überhaupt möglich ist, liegt m.E. am Korrelat insofern. Ohne Korrelat erzeugt Mittelstellung auch bei eigentlich integrierten Adverbialsätzen einen Desintegrationseffekt, sodass nicht mehr von echter Mittelfeld-Besetzung gesprochen werden kann; s. zu dieser Einschätzung allgemeiner Reich und Reis (2013: 557) sowie bestätigend mit Blick auf eigentlich klar verbbezogene hypothetische Vergleichssätze Bücking (2015: 277-278). Die durch einen eigenen Hauptakzent auf dem Matrixprädikat angezeigte prosodische Selbstständigkeit des Bezugssatzes in (26b)/ (27b) sorgt umgekehrt für die holistische SIA-Interpretation. Hier steht der Adverbialsatz nicht im Mittelfeld des Matrixsatzes; ich halte es mit Reich und Reis (2013) für plausibel, dass solche Binnenstellungen als Parenthesen zu analysieren sind (s. die graphische Auszeichnung durch Gedankenstriche).

Auch der Befund für das Vorfeld ist im Wesentlichen gleich, s. dazu die Paare in (28) und (29).

(28) a. In/SOfern, als sie GÜN\stig ist, ist die Lösung attraktiv.

b. In/SOfern, als sie /GÜNstig ist, ist die Lösung attrakTIV\.

(29) a. In/SOfern, als er geSUND $\backslash$ ist, geht es Ben gut.

b. In/SOfern, als er ge/SUND ist, geht es Ben GUT\. 
Wie vorausgesagt, erlauben die Beispiele in (28a)/(29a) ohne separaten Hauptakzent im Matrixsatz nur die LIA-Interpretation, während ein separater Hauptakzent wie in (28b)/(29b) zur SIA-Interpretation führt. Um diese Differenzierung adäquat einzuordnen, möchte ich drei Aspekte kommentieren.

Erstens ist bemerkenswert, dass sich der prosodische Unterschied selbst bei anaphorischem Gebrauch nachweisen lässt; s. dazu folgende Dialogvarianten:

(30) A: Mein zukünftiger Ehemann hat reich geerbt.

a. B: Aha, in/SOfern\ ist er als Ehemann attraktiv. Ich hatte mich auch schon gewundert; sonst ist er ja nicht Dein Typ.

b. B: Klar, in/SOfern ist er als Ehemann attrakTIV \. (\#Ich hatte mich auch schon gewundert; sonst ist er ja nicht Dein Typ.)

Wenn - wie in (30a) - allein insofern die Hutkontur bildet und das Matrixprädikat keinen eigenen Hauptakzent erhält, schränkt die aus der Vorgängeräußerung erschlossene Hinsicht das Matrixprädikat lexikalisch ein. B kann somit explizit andere Attraktivitätsdimensionen (z. B. äußerliche Attraktiviät) ausschließen. B’s Antwort wirkt in diesem Fall ausgesprochen schnippisch. Wenn hingegen - wie in (30b) - das Matrixprädikat einen eigenen Hauptakzent erhält, deutet man B's Reaktion anders: B bestätigt hier, dass der Erbreichtum des Zukünftigen ausreicht, ihn als attraktiv $\mathrm{zu}$ bezeichnen; es liegt also eine SIA-Interpretation vor. Erwartungsgemäß korreliert diese Interpretation damit, dass der Ausschluss alternativer Hinsichten nicht unmittelbar zur Debatte steht (s. die Diskussion in Abschnitt2.1 oben). Die Kürze des einschränkenden Ausdrucks führt damit nicht automatisch zur prosodischen Integration; dies legt nahe, dass die prosodische Differenzierung tatsächlich ein Effekt der unterstellten lesartensensitiven Fügungsunterschiede ist.

Zweitens sei erwähnt, dass LIA-Sätze im Vorfeld generell informationsstrukturell auffälliger sind als SIA-Sätze. Dies erklärt sich aber genau dadurch, dass VP-interne Modifikatoren im Vorfeld nie informationsstrukturell neutral sind; demgegenüber ist sententialer Bezug besonders verträglich mit einer linear frühen Position. Eine entsprechende ausführliche Diskussion der (Pragmatik der) Vorfeldbesetzung im Deutschen liefert z. B. Frey (2006). Gut illustrieren lässt sich der Punkt anhand verschiedener Lokativtypen, s. die auf Maienborn (2001: [44]) aufbauenden Beispiele in (31) und (32). So verlangt der präfinite VP-interne Lokativ in (31) - lokalisiert werden Teile des Ereignisses, hier die Füße von Peter - einen variabel wählbaren engen Fokus. Der rahmensetzende Lokativ in (32) - es wird eine Aussage über Peters Aufenthaltszeit im Auto gemacht erzwingt hingegen bei Voranstellung keinen engen Fokus; der finale Hauptakzent kann also als fokusprojizierender neutraler Akzent gedeutet werden. 
(31) a. In STIEfeln hat Peter geschlafen (nicht in PanTOFfeln).

b. In Stiefeln hat PEter geschlafen (nicht BEN).

c. In Stiefeln hat Peter geSCHLAfen (nicht geTANZT).

(32) Im Auto hat Peter geSCHLAfen.

Bereits in Abschnitt2.3 wurde illustriert, dass LIA-Sätze neben dem engen Fokus auf dem Adverbialsatz als Alternative auch einen engen Fokus auf dem Subjekt erlauben, s. (20b) oben sowie als weitere Kandidaten die Beispiele in (33).

(33) a. Insofern, als sie günstig ist, ist die WIEner Lösung attraktiv (nicht die BREmer).

b. Insofern, als er verliebt ist, geht es BEN gut (nicht PEter).

Diese Beispiele sind dann legitim, wenn die jeweiligen dimensional spezifizierten Prädikationen kontextuell bereits eingeführt sind und zur Diskussion steht, wer sie erfüllt. Charakteristisch ist jeweils, dass die LIA-Sätze nur phrasale Akzente und keinen eigenen Hauptakzent tragen. Da m. E. auch hier keine SIAInterpretation möglich ist, bestätigen auch diese Beispiele eine Korrelation zwischen LIA-Interpretation und einteiliger Fokus-Hintergrund-Gliederung. ${ }^{7}$

Drittens: Auf den ersten Blick mag überraschen, dass SIA-Sätze überhaupt in linksadjazenter präfiniter Position stehen können. Denn identifiziert man diese Position mit dem Vorfeld und versteht darunter eine notwendige Integrationsposition, läge damit ein starkes Argument gegen die Desintegrationsanalyse

7 Erinnert sei allerdings an das in Fußnote 4 angesprochene inhärente Interpretationsproblem für solche Fälle. Marga Reis hat mich gefragt, ob auch enger Fokus auf dem Matrixprädikat möglich ist. Ein entsprechendes Beispiel könnte (i) sein, s. auch analog mit adjektivischer dimensionaler Beschränkung (ii). Dabei müsste man einen Kontext annehmen, in dem die Hinsicht gegeben ist und zur Debatte steht, welches lexikalische Matrixprädikat sie dimensional spezifiziert.

(i) Insofern, als er verliebt ist, fühlt sich Ben KOmisch, nicht GUT.

(ii) Gefühlsmäßig fühlt sich Ben KOmisch, nicht GUT.

Ich bin unsicher, ob die Beispiele tatsächlich entsprechende LIA-Lesarten haben können. Mein Eindruck ist eher, dass man auf SIA-Interpretationen ausweicht. Dies läuft für z. B. (i) auf folgende Interpretation hinaus: Relativ zur Tatsache, dass Ben verliebt ist, kann man sagen, dass er sich komisch fühlt, nicht, dass er sich gut fühlt. Dieser Eindruck könnte damit zusammenhängen, dass mit der Betonung auf dem Matrixprädikat automatisch seine holistische Interpretation zur Debatte steht oder zumindest verunklart wird, dass der Matrixpart gerade keine separate Fokus-Hintergrund-Gliederung bilden sollte. Details dieser möglichen Kontamination der LIA-Interpretation sind mir aber unklar; ich lasse diesen Punkt hier offen. 
von SIA-Sätzen vor. Allerdings hat die Literatur in den letzten Jahren gezeigt, dass einige weitere Adverbialsatztypen präfinit möglich sind, obwohl sie nach Ausweis anderer (Des)Integrationstests als desintegriert oder zumindest nicht vollständig integriert $\mathrm{zu}$ gelten haben, s. insbesondere Reis und Wöllstein (2010), Frey (2011) und Christ (2014). Zu den prominenten Beispielen gehören V1-Konditionale sowie adversative während-Sätze, s. (34):

(34) a. Wäre ich MillioNÄR, würde ich es TUN.

(Reis und Wöllstein 2010: [63'c])

b. Während PEter JUbelte, war sein BRUder eher entTÄUSCHT.

(Reis und Wöllstein 2010: [80a])

Für mein Hauptanliegen hier ist zunächst nur wesentlich, dass sich SIA-Sätze in dieses Muster einreihen und deshalb ihr unmarkiertes Vorkommen in präfiniter Position wie in (28b)/(29b) kein Indiz für (vollständige) Integration liefert. Aus Platzgründen kann ich hier nicht ausführlich diskutieren, ob man die entsprechenden desintegrierten Sätze besser als so genannte periphere Adverbialsätze in SpecC und damit dem Vorfeld des Bezugssatzes analysiert (s. Frey 2011) oder ob man sie als linksseitige CP-Adjunkte analysieren sollte und sich damit die Konsequenz einhandelt, dass das Vorfeld des Bezugssatzes elliptisch oder ganz unbesetzt ist (s. Reis und Wöllstein 2010). ${ }^{8}$ Ich möchte allerdings kurz auf folgenden Punkt der Kontroverse eingehen, auf den mich Marga Reis aufmerksam gemacht hat: Reis und Wöllstein (2010) nutzen als zentrales Indiz für ihre Analyse die prosodische Eigenständigkeit von sowohl V1-Konditionalen als auch adversativen während-Sätzen in präfiniter Position, s. die entsprechende prosodische Markierung der Beispiele in (34). Brisant ist zudem, dass es bei den adversativen Sätzen (anders als bei V1-Konditionalen) kein plausibles Resumptiv gibt, s. (35), also die Annahme einer elidierten Proform unmotiviert bleibt. Damit liefern adversative Gefüge prima facie gute Evidenz dafür, dass das Deutsche die Kombination aus einem linksseitigen desintegrierten Adverbialsatz mit einem V1-Deklarativ als Bezugssatz regelhaft erlaubt.

(35) Während PEter JUbelte, ( ${ }^{\star}$ so, ${ }^{\star} d a,{ }^{\star}$ dann) war sein BRUder eher entTÄUSCHT.

(Reis und Wöllstein 2010: [82a])

8 Nach Frey (2011) sind periphere Adverbialsätze nicht desintegriert, sondern semi-integriert; ich verzichte hier auf diese Differenzierung. Klar sollte ebenso sein, dass man gemäß der Analyse von Reis und Wöllstein (2010) erst gar nicht von SIA-Sätzen im Vorfeld sprechen kann. Der Begriff „Vorfeld“ ist also in diesem Artikel lax zu verstehen; er bezeichnet theorieneutral die Position, die an der Oberfläche linksadjazent zum finiten Verb erscheint. 
Christ (2014: 236-237) verweist allerdings darauf, dass adversative währendGefüge notwendig einen Kontrast zwischen Bezugssatz und abhängigem Satz involvieren und dieser an einen je eigenen Kontrastfokus geknüpft ist. Er argumentiert (aufbauend auf der analogen Deutung von je-desto-Gefügen in Speyer [2011]), dass der separierte Hauptakzent damit ein plausibles alternatives Motiv hat und nicht mehr auf die angenommene Desintegration des adversativen Satzes zurückgeführt werden muss. Vor diesem Hintergrund sind nun aber SIASätze m. E. deshalb aufschlussreich, weil sie ebenfalls prosodisch desintegriert sind, dies aber nicht an einer Kontrast-Relation zwischen abhängigem Satz und Bezugssatz liegen kann: Die vom als-Satz gelieferte Hinsicht steckt den Rahmen für die Evaluation des Bezugssatzes ab, kontrastiert aber nicht mit ihm; unerheblich ist dabei, dass ein Kontrast $\mathrm{zu}$ anderen (kontextuell gegebenen oder erschließbaren) Hinsichten besteht. Gäbe es einen semantisch-pragmatisch vermittelten fügungsinternen Kontrast, müsste dieser zudem auch für LIA-Sätze gelten, die aber ja gerade mit nur einer Fokus-Hintergrund-Gliederung auskommen. Der Befund unterstreicht, dass die prosodische Eigenständigkeit von SIASätzen als strukturell relevantes Indiz ernst zu nehmen ist; die naheliegende Schlussfolgerung lautet, sie als desintegrierte CP-Adjunkte $\mathrm{zu}$ behandeln. Gleichzeitig liefern SIA-Gefüge ein unabhängiges Argument für die These in Reis und Wöllstein (2010), dass es präfinite Adverbialsätze gibt, die nicht im Vorfeld des Bezugssatzes stehen. Da SIA außerdem keine Resumptiva zu erlauben scheinen, s. (36), taugen sie vielleicht sogar als Evidenz für eine Analyse der entsprechenden Bezugssätze als V1-Deklarative.

(36) In/SOfern, als sie / GÜNstig ist, $\left({ }^{\star}\right.$ so, ${ }^{\star}$ da, ${ }^{\star}$ dann) ist die Lösung attrakTIV\.

\subsection{Variablenbindung}

Variablenbindung aus einem Matrixsatz in einen abhängigen Satz setzt voraus, dass der abhängige Satz im c-Kommando-Bereich der bindenden Konstituente des Matrixsatzes steht, also in den Matrixsatz integriert ist. In LIA-Gefügen kann ein Quantor in Subjektposition ein Personalpronomen im abhängigen Satz binden; s. die Stellungsvarianten in (37) mit der für LIA-Gefüge charakteristischen Prosodie und Interpretation:

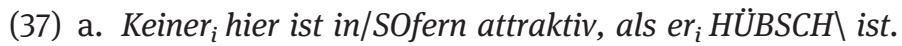

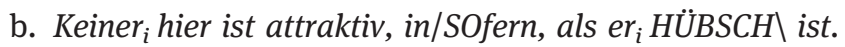

,Für niemanden gilt, dass er in einer Hinsicht attraktiv ist, die sich aus seiner Hübschheit ergibt.‘ 
Die satzinterne Rekonstruierbarkeit zeigt sich ebenfalls bei Voranstellung des abhängigen Satzes. So liegt auch in den Beispielen (38) die LIA-Interpretation vor, wobei analog zu den Befunden in den Abschnitten 2.3 und 2.4 mit den jeweils gewählten Fokusexponenten unterschiedliche informationsstrukturelle Effekte einhergehen:

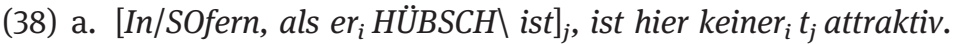
,Zur Debatte steht, in welcher Hinsicht keiner attraktiv ist.

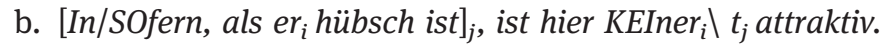
,Zur Debatte steht, wer äußerlich attraktiv ist.‘

SIA-Gefüge hingegen verbieten Variablenbindung, und zwar unabhängig von der jeweils gewählten Stellungsvariante, s. (39). Am deutlichsten ist der Befund m. E. für die Variante (39b), da hier weder der mit dem Korrelat einhergehende Progredienzeffekt noch die mit dem Vorfeld einhergehenden Deutungskomplikationen interferieren. Die Explikation der intendierten SIA-Lesart ist jeweils schwierig. Ob man aber für die als-Proposition auf alle oder niemand zurückgreift (was je nach Negationsbezug die Optionen wären), ist egal - beide Lesarten sind nicht möglich.

(39) a. * Keiner $_{i}$ hier ist in/SOfern attraktiv, als er $_{i} / \mathrm{HÜBSCH}$ ist $\backslash$.

b. * Keiner $_{i}$ hier ist attrakTIV\, in/SOfern, als er $/$ HÜBSCH ist $\$.

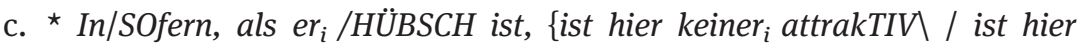
KEIner $_{i} \backslash$ attraktiv\}.

,In Hinsicht darauf, dass für niemanden/jeden gilt, dass er hübsch ist, kann man sagen, dass niemand attraktiv ist.

Lizenziert ist hier allenfalls eine ungebundene Interpretation: Weil eine bestimmte kontextuell gegebene Person hübsch ist, kann man sagen, dass hier niemand attraktiv ist. Dies setzt einen sehr spezifischen Kontext voraus (es muss eine äußerlich klar als besonders hübsch hervorstechende Person geben; der Sprecher muss diese zum Vergleichsstandard für eine holistische Evaluation machen), zeigt aber vielleicht dennoch, dass die Sätze nicht als solche ungrammatisch sind, sondern ein Indiz dafür liefern, dass die SIA-Interpretation mit Bindung inkompatibel ist.

Der Effekt lässt sich mit positivem Quantor replizieren, s. den Kontrast in (40). Während die LIA-Interpretation in (40a) Bindung erlaubt, ist die holistische SIA-Interpretation in (40b) bindungsresistent. Beachtenswert ist dabei, dass die SIA-Interpretation hier konzeptuell durchaus Sinn macht, aber dennoch klar ausgeschlossen ist. Zum Ausdruck der holistischen Interpretation 
muss man wie in Beispiel (41) auf die Wiederholung des Quantors statt auf Etablierung einer Bindungsrelation zurückgreifen. ${ }^{9}$

(40) a. Jede Lösung hier ist attraktiv, in/SOfern als sie ${ }_{i}$ GÜNstig $\backslash$ ist.

,Für jede Lösung gilt, dass sie in einer Hinsicht attraktiv ist, die sich aus ihrem niedrigen Preis ergibt.

b. * Jede Lösung hier ist attrakTIV\, in/SOfern als sie $_{i} /$ GÜNstig ist $\$. ,In Hinsicht darauf, dass für jede Lösung gilt, dass sie günstig ist, kann man sagen, dass jede Lösung attraktiv ist.‘

(41) Jede Lösung hier ist attrakTIV\, in/SOfern als jede Lösung hier / GÜNstig ist \.

\subsection{Zwischenfazit}

Gefüge auf der Basis von insofern als kommen in zwei Strukturvarianten vor: LIA-Sätze sind strukturell in ihren Bezugssatz integriert; entsprechend stehen sie im Skopus von Negation und Fokuspartikeln, projizieren innerhalb lexikalischer Phrasenkategorien, bilden mit dem Matrixsatz eine Fokus-HintergrundGliederung und erlauben Variablenbindung. SIA-Sätze hingegen beziehen sich auf den Bezugssatz als Ganzen und sind als solche strukturell desintegriert; so skopieren sie oberhalb von Negation und Fokuspartikeln, verbieten die Projek-

9 Es läge nahe, das Bindungsverhalten auch anhand von Prinzip-C-Effekten zu überprüfen, sich also zunutze zu machen, dass gemäß Bindungsprinzip C selbstständig referierende Eigennamen bzw. definite Kennzeichnungen nicht von referenzidentischen Ausdrücken c-kommandiert werden dürfen. Die Dateneinschätzung ist allerdings aufgrund der Vielzahl interferierender Faktoren noch subtiler als im Fall von Variablenbindung. Benannt sei deshalb nur kurz, wie ein solcher Kontrast aussehen könnte. Nach Reis und Wöllstein (2010: 144-145) erlauben z. B. nachgestellte V1-Konditionale, nicht aber ihre wenn-Pendants Koreferenz, s. (i). Die Autorinnen werten diesen Kontrast als Indiz dafür, dass V1-Konditionale desintegriert, wenn-Konditionale hingegen integriert sind. M.E. stimmt die Einschätzung, sie setzt allerdings die Ausblendung der gegenläufigen Linearisierungspräferenz voraus.

(i) a. * Sie ${ }_{i}$ ruft nach Hans, wenn Maria ${ }_{i}$ hungrig ist. (Reis und Wöllstein 2010: [56a])

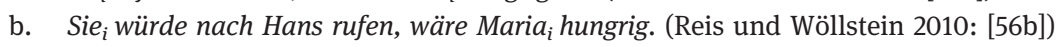

M.E. liefern nachgestellte IA-Sätze ein analoges Bild: LIA-Gefüge zeigen einen Prinzip-CEffekt, s. (iia), sind also integriert. SIA-Gefüge hingegen erlauben - erneut unter Ausschaltung der gegenteiligen Linearisierungspräferenz - Koreferenz, s. (iib), was als Indiz für Desintegration gelten kann.

(ii) a. * Es geht ihm ${ }_{i}$ gut, in/SOfern als Ben ${ }_{i}$ geSUND $\backslash$ ist.

b. Es geht ihm ${ }_{i}$ GUT\, in/SOfern als Ben ${ }_{i}$ ge/SUND ist $\backslash$. 
tion innerhalb lexikalischer Kategorien, haben notwendig eine eigene FokusHintergrund-Gliederung und lassen keine Variablenbindung zu.

Wie eingangs erwähnt geht es mir hier um keine vollständige syntaktische Analyse von IA-Gefügen. Die Befunde erlauben aber den Rückschluss auf eine kompositional unterschiedliche Ausgangsposition für LIA- vs. SIA-Gefüge. So hat Reis (1997) (s. auch Reis und Wöllstein 2010) dafür argumentiert, dass integrierte Nebensätze direkt von der verbalen Matrixprojektion lizenziert und damit als deren integraler Bestandteil ausgewiesen werden. Demgegenüber können desintegrierte Nebensätze als CP-Adjunkte analysiert werden, die entsprechend nicht in ihre Bezugssätze eingebettet sind. Vor diesem Hintergrund halte ich es für gut gerechtfertigt, dass LIA-Gefüge Zugriff auf die lexikalischen Binnenkomponenten des Matrixsatzes haben, SIA-Gefüge hingegen auf die von der MatrixCP bereitgestellte Proposition als Ganze zielen.

\section{Zur Dimensionssensitivität von IA-Gefügen}

Die obige Strukturanalyse legt nahe, dass LIA-Gefüge Dimensionen lexikalischer Prädikationen und SIA-Gefüge Dimensionen von Situationsbeschreibungen als Ganzen ins Spiel bringen. Dieser Abschnitt widmet sich der angenommenen Dimensionssensitivität aus semantisch-pragmatischer Perspektive. Das Ziel ist dabei, den invarianten Bedeutungskern von IA-Gefügen zu bestimmen und gleichzeitig die Grundlage für eine Ableitung der strukturabhängigen Effekte $\mathrm{zu}$ schaffen. In Abschnitt3.1 geht es um die Rolle lexikalisch angelegter (Mehr)Dimensionalität für die Bedeutungskonstitution von IA-Gefügen; in Abschnitt 3.2 wird das Verhältnis zwischen dimensionaler Spezifizierung und der (subjektiven) Auswahl von „Interpretationen“ diskutiert. Als wesentliche Kontrastfolie dient jeweils der Vergleich mit dem Verhalten des subjektiven Einstellungsverbs finden.

\subsection{IA-Gefüge und lexikalisch angelegte (Mehr)Dimensionalität}

In der Literatur wird traditionell zwischen mehrdimensionalen und eindimensionalen lexikalischen Prädikaten unterschieden, s. zu einer detaillierten Diskussion Sassoon (2013a) und Sassoon (2013b). Mit z. B. Sassoon (2013a: 235-236) kann man den zentralen Unterschied wie folgt beschreiben: Mehrdimensionale Prädikate wie ähnlich, gesund oder gut können gleichzeitig mit verschiedenen Dimensionen assoziiert werden; so kann z. B. ähnlich in Hinsicht auf Farbe oder 
Form interpretiert werden. Demgegenüber werden eindimensionale Prädikate wie schwer oder lang mit nur einer (skalar interpretierten) Dimension verknüpft. $\mathrm{Zu}$ beachten ist, dass Mehrdimensionalität auf der gleichzeitigen Assoziierbarkeit verschiedener Dimensionen beruht und nicht mit einer Ambiguität zwischen separaten Dimensionen zu verwechseln ist. Diesen Punkt illustriert Sassoon anhand des Kontrasts in (42). Das mehrdimensionale Prädikat identical kann mit Hilfe einer with respect to-Phrase dimensional eingeschränkt werden; diese Art der Einschränkung kann aber nicht die Auswahl zwischen temporaler vs. spatialer Interpretation des ambigen Prädikats long steuern.

(42) a. The boxes are identical with respect to size and weight / in all respects. (Sassoon 2013a: [1a]/[2a])

b. \# The wedding is long with respect to temporal duration (but not with respect to space) / in all respects.

(Sassoon 2013a: [1c])

Im Folgenden soll nun geklärt werden, wie sich die Unterscheidung zwischen mehrdimensionalen vs. eindimensionalen Prädikationen auf die Interpretation und Distribution von IA-Gefügen auswirkt.

\subsubsection{IA-Gefüge und lexikalisch angelegte Mehrdimensionalität}

Die bisherigen Überlegungen sagen voraus, dass LIA-Gefüge dann lizenziert sind, wenn die VP des Matrixsatzes eine mehrdimensionale und damit dimensional spezifizierbare Prädikation liefert. Dies passt dazu, dass LIA-Gefüge problemlos in Kombination mit mehrdimensionalen Prädikaten wie evaluativen Prädikaten oder Geschmacksprädikaten auftreten können, s. die Beispiele in Abschnitt 2 auf der Basis der Prädikate attraktiv bzw. gut geh- sowie die Beispiele in (43) und (44).

(43) a. Insofern schön, als die Blumen farblich zueinander passen, ist PAULS, nicht aber HANnos Blumenstrauß.

b. Keiner ${ }_{i}$ hier ist in/SOfern kompetent, als er ${ }_{i}$ die logischen GRUND\lagen durchschaut.

(44) Die Suppe ist nur in/SOfern lecker, als sie fein geWÜRZT \ist. Hingegen erzeugt der doch recht holzige Rosmarin ein unangenehmes Mundgefühl.

Die gegebene Bedingung wird auch dann erfüllt, wenn eine mehrdimensionale Prädikation lexikalisch angelegt ist, sich aber erst aus der Kombinatorik der 
verschiedenen VP-Komponenten kompositional ergibt. Beispiele für diese Lizenzierung liefern Vergleichskonstruktionen wie in (45). Wie erwartet sind auch hier LIA-Gefüge wohlgeformt. ${ }^{10}$

(45) a. Bella ähnelt nur in/SOfern ihrer Tochter, als sich ihre NA\sen gleichen. Ansonsten sind sie sehr verschieden.

b. Bella wirkte nur in/SOfern alt, als sie ganz eingefallene AU\gen hatte. Ihre Stimme hingegen war kräftig und voller Elan.

Der LIA-Satz muss dabei weiten Skopus über den Vergleich haben. Bestätigung dafür liefert die Beobachtung, dass bei einer komplexen Verschachtelung von LIA-Satz und z. B. modal interpretiertem hypothetischem Vergleichssatz wie in (46) der Vergleichssatz verbnäher projiziert und damit der kompositionalen Integration des LIA-Satzes vorangeht. ${ }^{11}$

(46) a. Bella ist insofern Rad gefahren, als wäre sie betrunken, als sie Trunkenheit simulierte.

b. * Bella ist insofern Rad gefahren, als sie Trunkenheit simulierte, als wäre sie betrunken.

Die genannten mehrdimensionalen Prädikationen sind ebenfalls in SIA-Gefügen möglich, s. die Beispiele in (47a) und (47b). Als formales Kennzeichen für SIA-Status dient hier jeweils eine separierte Fokus-Hintergrund-Gliederung.

(47) a. In/SOfern, als sie fein ge/WÜRZT ist, ist die Suppe LEC \ker. (\#Ansonsten ist sie aber nicht lecker.)

b. In/SOfern, als sich ihre /NAsen gleichen, ÄH\nelt Bella ihrer Tochter. (\#Ansonsten ähneln sie sich aber kaum.)

Mit dem SIA-Status korreliert regulär, dass hier die als-Satz-Proposition die Matrixaussage als Ganze rechtfertigt. Wie in Abschnitt 2.1 diskutiert kann eine einzelne Hinsicht als hinreichend für die holistische Evaluation mehrdimensionaler Prädikationen konzipiert werden; systematischer Reflex dieser Akkommodation ist, dass alternative Hinsichten als irrelevant erscheinen.

Bemerkenswert ist, dass die genannten Prädikatsgruppen typischerweise auch unter finden einbettbar sind, s. dazu Sæbø (2009), Reis (2013a) und Um-

10 Diese Beispiele bauen auf den parallelen Beispielen mit finden in Reis (2013a: [27]) auf.

11 Bücking (2017) diskutiert die V-Adjazenz modal interpretierter hypothetischer Vergleichssätze ausführlicher; den Hintergrund bildet dabei die bekannte Beobachtung, dass an der rechten Peripherie Subdomänen Superdomänen linear vorangehen. 
bach (2016). Dies leistet der eingangs erwähnten Annahme Vorschub, dass es sowohl bei IA-Gefügen als auch bei finden um subjektive Einschätzungen urteilersensitiver Prädikationen geht. Ein erstes Indiz gegen diese Annahme liefern die Beispiele in (48), die auf dem mehrdimensionalen, aber (in „objektiver“ Lesart) von einem Urteiler unabhängigen Prädikat krank basieren.

(48) a. \# Ich finde Paul krank. Er hat einen grippalen Infekt.

b. Paul ist in/SOfern krank, als er einen grippalen InFEKT $\backslash$ hat.

Während krank die Einbettung unter finden verbietet, ist es in LIA-Gefügen grammatisch. Der Kontrast deutet darauf, dass LIA-Gefüge dimensions-, nicht aber urteilersensitiv sind. Mit Blick auf evaluative Prädikate, Geschmacksprädikate sowie Vergleichskonstruktionen ist der Unterschied nur deshalb nicht sichtbar, weil diese sowohl mehrdimensional als auch urteilersensitiv sind.

\subsubsection{IA-Gefüge und eindimensionale Prädikate}

Die Beispiele in (49)/(50) zeigen, dass LIA-Gefüge inkompatibel mit eindimensionalen Prädikaten wie schwer oder kurz sind. Dies liefert eine zentrale bestätigende Evidenz für die Hypothese, dass LIA-Gefüge eine mehrdimensionale Prädikation als Bezugsgröße voraussetzen.

(49) a. \# Ben ist nur in/SOfern schwer, als er hundert KI\lo wiegt.

b. \# Ben ist ein in/SOfern schwerer Mann, als er hundert KI\lo wiegt.

c. \# Keiner ${ }_{i}$ hier ist in/SOfern schwer, als er ${ }_{i}$ hundert KI\lo wiegt.

(50) a. \# In/SOfern kurz, als es nur eine halbe STUN\de dauerte, war das Tennisspiel.

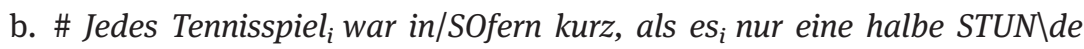
dauerte.

c. \# Das Tennisspiel dauerte nur eine halbe Stunde. In/SOfern \war es kurz.

Für SIA-Gefüge ist die Situation eine andere. Da sie spezifizieren, in welcher Hinsicht eine Aussage als Ganze gerechtfertigt ist, sollten sie nicht sensitiv bezüglich einer lexikalisch angelegten Mehrdimensionalität sein. Bildet man zu den obigen Beispielen SIA-Pendants, s. (51)/(52) mit jeweils separierten FokusHintergrund-Gliederungen, bestätigt sich, dass eindimensionale Prädikate in SIA-Gefügen regulär möglich sind. Die jeweilige Voraussetzung ist ein passender etablierter Zusammenhang; so liefert ein Körpergewicht von $100 \mathrm{~kg}$ einen 
guten Grund, jemanden als schwer zu bezeichnen, und ein halbstündiges Tennisspiel kann als kurz gelten.

(51) a. Ben ist in/SOfern schwer, als er hundert /KIlo wiegt $\$.

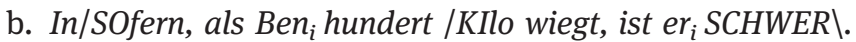

(52) a. In/SOfern, als das Tennisspiel ${ }_{i}$ nur eine halbe /STUNde dauerte, war es ${ }_{i}$ KURZ\.

b. Das Tennisspiel dauerte nur eine halbe Stunde. In/SOfern war es KURZ\.

Ein weiteres Indiz dafür, dass LIA-Gefüge den Bezug auf eine mehrdimensionale Prädikation präsupponieren, liefert Negation. LIA-Gefüge erlauben lexikalische Negation mit engem Skopus (Kontraritäten), s. (53), nicht aber Satznegation mit engem Skopus (Kontradiktionen), s. (54). ${ }^{12}$

(53) a. Jeder ${ }_{i}$ hier ist in/SOfern unzuverlässig, als er immer UN\pünktlich ist. (Jeder $_{i}$ ist aber insofern zuverlässig, als er ${ }_{i}$ seine Aufgaben immer gewissenhaft erledigt.)

b. Ben ist nur in/SOfern unzuverlässig, als er immer UN\pünktlich ist. (Ansonsten erledigt er alle Aufgaben sehr gewissenhaft.)

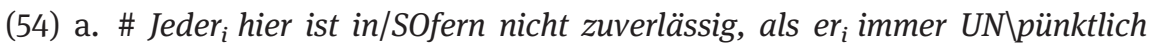
ist. (Jeder ${ }_{i}$ ist aber insofern zuverlässig, als er r $_{i}$ seine Aufgaben immer gewissenhaft erledigt.)

b. \# Ben ist nur in/SOfern nicht zuverlässig, als er immer UN〉pünktlich ist. (Ansonsten erledigt er alle Aufgaben sehr gewissenhaft.)

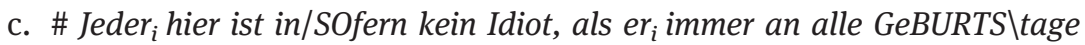
denkt.

Dieser Befund lässt sich vor dem Hintergrund der angenommenen Restriktion gut unabhängig motivieren: Die lexikalische Negation in (53) bildet ein mehrdimensionales Prädikat auf ein mehrdimensionales Antonym ab; deshalb sind hier LIA-Gefüge unauffällig. Demgegenüber führt Satznegation wie in (54) zu einem ontologisch abstrakten Zustand ohne dimensionale Spezifizierungsoption (s. zum Verhältnis von Zuständen und Abschluss unter Negation u. a. Maienborn [2005], Bücking [2012]). Dies sagt korrekt voraus, dass LIA-Gefüge hier markiert sind.

Eine offene Frage ist, ob man lexikalisch nicht vorgesehene Mehrdimensionalität akkommodieren kann. Relevante Beispiele sind in (55) und (56) gegeben,

12 S. Abschnitt 2.2 oben zu SIA-Gefügen bzw. weitem Negationsskopus. 
in denen die jeweiligen als-Sätze alternative „Dimensionen“ von Weite ( \pm Luftlinie) sowie Teure $( \pm$ direkte Kosten) nahelegen.

(55) a. ? Der Kaffee in Restaurant $A$ ist in/SOfern teuer, als man dort auch für den SITZ\platz zahlt.

b. Der Kaffee in Restaurant $A$ ist in/SOfern teuer, als man dort auch für den /SITZplatz zahlt $\backslash$.

(56) a. ? In/SOfern, als man um den See heRUM\fahren muss, ist der Weg zur Schule weit.

b. In/SOfern, als man um den See he/RUMfahren muss, ist der Weg zur Schule WEIT\.

Wie erwartet, sind die jeweiligen SIA-Gefüge (55b) und (56b) unabhängig von der Akkommodationsfrage grammatisch. Für die LIA-Pendants ist die Lage m. E. nicht eindeutig: Einerseits halte ich die Beispiele (55a) sowie (56a) nach wie vor für markiert; andererseits führt die nahegelegte Akkommodationsoption dazu, dass die Beispiele akzeptabler sind als die LIA-Gefüge in (49) und (50) oben. Nun ist bekannt, dass Präsuppositionen oft akkommodiert werden können. Plausibel ist zudem, dass es sich bei \pm mehrdimensional letztlich um eine konzeptuell motivierte Distinktion handelt, die sich entsprechend an pragmatisch vermittelte Bedingungen anpassen kann. Eine genauere Erfassung dieser Flexibilität (und ihrer Grenzen) möchte ich hier offenlassen.

Betont sei abschließend, dass eindimensionale Prädikate unter finden einbettbar sind, s. (57).

(57) Ich finde $\{$ Ben schwer / das Spiel kurz / den Kaffee teuer\}.

In der Literatur wird kontrovers diskutiert, was die Quelle für diese Option ist und welchen Restriktionen sie unterliegt, s. vor allem Sæbø (2009), Kennedy (2013) und Umbach (2016). Wesentlich für die Argumentation hier ist lediglich, dass sich finden erneut klar anders als LIA-Gefüge verhält, also ein weiteres Indiz gegen eine analoge Behandlung ihrer jeweiligen Interpretation vorliegt.

\subsection{IA-Gefüge und das Verhältnis zwischen dimensionaler Spezifizierung und der (subjektiven) Auswahl von „Interpretationen“}

Im Einklang mit der bisherigen Argumentation dienen IA-Gefüge dazu, Matrixprädikationen durch die Relativierung auf Hinsichten dimensional zu spezifi- 
zieren und damit auf eine in bestimmter Weise eingeschränkte Interpretation festzulegen; in diesem Sinne besteht der metasprachliche Effekt von IA-Gefügen darin, Prädikationen in dimensional spezifizierte Prädikationen zu überführen. In der Literatur ist der Begriff „Interpretation“ ganz wesentlich für verschiedene andere Strukturen genutzt worden; dies wirft die Frage auf, wie sich IA-Gefüge zu diesen Strukturen verhalten. Im Folgenden zeige ich, dass der für IA-Gefüge relevante Interpretationsbegriff nicht mit diesen Vorbildern zusammenfällt. Dies bestätigt indirekt, dass sich der metasprachliche Effekt von IAGefügen tatsächlich aus ihrer spezifischen Dimensionssensitivität ableitet.

\subsubsection{IA-Gefüge und die Auswahl von „Interpretationen“ für Prädikate}

Eine Möglichkeit, Prädikate auf bestimmte Interpretationen zu beschränken, besteht darin, für sie Satisfaktionskriterien festzulegen, s. Barker (2002) und Krifka (2013). Diese Option ist typisch für vage Prädikate wie z. B. groß. So dienen die Strukturen in (58) nicht dazu, auf der Basis einer für groß präsupponierten Interpretation Aussagen über die Welt zu machen. Stattdessen werden umgekehrt Bedingungen für die Interpretation eingeführt. Mit (58a) wird ein numerisches Maß als Mindestanforderung expliziert und mit (58b) wird Bens Größe zum relevanten Maßstab gemacht.

(58) a. [Kontext: Zur Diskussion steht, wie groß zu interpretieren ist.] Groß ist, wer $1,90 \mathrm{~m}$ groß ist.

b. [Kontext: Zur Diskussion steht, wie groß zu interpretieren ist; Größe von Ben ist bekannt.]

$B E N$ ist groß.

Diese Art Interpretationsfestlegung ist für LIA-Gefüge ausgeschlossen, s. (59). Erinnert sei ebenso an die in Abschnitt 3.1.2 diskutierte Beobachtung, dass eindimensionale und ebenfalls vage Prädikate wie schwer oder kurz mit LIA-Lesarten inkompatibel sind.

(59) a. \# Ben ist in/SOfern groß, als er einsNEUN\zig groß ist. [Hiermit lege ich gro $\beta$ auf die Interpretation $, \geq 1,90 \mathrm{~m}$ ' fest.]

b. \# Ben ist in/SOfern groß, als er größer als PAUL \ist. [Hiermit lege ich groß auf die Interpretation ,mind. so groß wie Paul' fest.]

Dieser Befund wird auch nicht dadurch in Frage gestellt, dass korrespondierende SIA-Gefüge grammatisch sind. Im Gegenteil: Ihre Akzeptabilität beruht gera- 
de darauf, dass sie auf vorausgesetzten Interpretationen des vagen Prädikats als Rechtfertigungen für die gemachten Aussagen aufbauen, s. dazu die Beispiele in (60) (mit den relevanten vorausgesetzten Interpretationsbedingungen für groß in Klammern) sowie - erneut analog - die Diskussion zu eindimensionalen Prädikaten oben.

(60) a. Ben ist in/SOfern groß, als er eins/NEUNzig groß ist $\$. [Und groß ist ja, wer $1,90 \mathrm{~m}$ groß ist.]

b. Ben ist in/SOfern groß, als er größer als /PAUL ist $\backslash$. [Und Paul ja groß ist.]

Einen ähnlichen Befund liefern ambige Prädikate. Die Auswahl einer Interpretation besteht in diesem Fall nicht in der Festlegung von Satisfaktionskriterien, sondern in der Festlegung auf eine Lesart. Auch diese Form der Interpretationsauswahl können LIA-Gefüge nicht leisten. So ist die Gegenüberstellung potenziell disambiguierender als-Propositionen wie in (61) abweichend.

(61) a. \# Dirk ist in/SOfern eine Ratte, als er uns verRA \ten hat, nicht aber in/SOfern, als er ein NA \getier ist.

b. \# Kein Tal ist in/SOfern weit, als es sehr BREIT ist; alle sind in/SOfern weit, als sie noch $100 \mathrm{~km}$ entFERNT $\backslash$ sind.

Analoge SIA-Sätze sind erneut grammatisch, s. (62). Allerdings gilt wie für vage Prädikate, dass Sprecher und Adressat jeweils eine Interpretation als die relevante voraussetzen können. So wird mit (62a) begründet, warum man Dirk als Ratte bezeichnen kann. Die Äußerung dient aber nicht dazu, die Interpretation von Ratte auf ,gemeiner Mensch' festzulegen, sondern setzt diese Interpretation als die zu begründende voraus. Entsprechend bleibt die Tier-Interpretation im gegebenen Zusammenhang von vornherein außen vor.

(62) a. Dirk ist in/SOfern eine Ratte, als er uns ver/RAten hat $\backslash$.

b. In/SOfern, als es noch $100 \mathrm{~km}$ ent/FERNT ist, ist das Tal WEIT\.

Damit bestätigt sich, dass LIAs sensitiv bezüglich Mehrdimensionalität im Sinne einer gleichzeitigen Assoziation verschiedener Dimensionen sind. Keine Rolle spielt hingegen Mehrdimensionalität im Sinne einer Ambiguität zwischen separaten Dimensionen, s. zur entsprechenden Unterscheidung die Einleitung zu Abschnitt 3.1.

Der metasprachliche Effekt von LIA-Gefügen liegt also weder darin, für Prädikate Satisfaktionskriterien festzulegen, noch darin, Prädikate zu disambiguie- 
ren. Stattdessen erhärtet sich die Annahme, dass LIA-Gefüge der dimensionalen Spezifizierung mehrdimensionaler lexikalischer Prädikationen dienen.

\subsubsection{SIA-Gefüge und die Auswahl von „Interpretationen“ für Sachverhalte}

Der Begriff „Interpretation“ spielt ebenfalls in der Analyse des Einstellungsverbs finden in Reis (2013a) eine zentrale Rolle. So argumentiert Reis dafür, dass finden eine subjektiv-epistemische Bedeutung einführt und diese auf sein Subjektargument $x$ sowie sein propositionales Argument $p$ in folgender Weise projiziert: $x$ wählt $p$ als eine „möglich[e] Interpretation eines zur Debatte stehenden Sachverhalts unter anderen möglichen Interpretationen“ (Reis 2013a: 417) aus. Das heißt für z. B. (63) im Wesentlichen Folgendes: Für einen präsupponierten Sachverhalt - nämlich eine bestimmte Größe von Ben - drückt der Sprecher aus, dass man diesen Sachverhalt seiner Meinung nach mit der Interpretation ,Ben ist groß` (nicht aber z. B. mit ,Ben ist klein') beschreiben kann.

\section{Ich finde, dass Ben groß ist.}

Betont sei, dass der Reis'sche Interpretationsbegriff damit ein grundlegend anderer ist als der bei Krifka (2013) oder Barker (2002): Bei Reis entsprechen Interpretationen den mit sprachlichen Ausdrücken verknüpften Propositionen; deshalb geht es hier um Interpretationen von Sachverhalten. Bei Krifka und Barker sind Interpretationen hingegen Kriterien zur adäquaten Verwendung sprachlicher Ausdrücke; es geht also um Interpretationen von sprachlichen Ausdrücken. ${ }^{13}$

Wie in der Einleitung in Anlehnung an Reis (2013a: Anm. 37) angedeutet haben SIA-Gefüge wie (64) (s. [60a] oben) auf den ersten Blick eine verwandte

13 Der metasprachliche Effekt von finden-Strukturen ist in der Analyse nach Reis (2013a) so auch lediglich indirekt: Indem die mit einem sprachlichen Ausdruck korrelierte Proposition als Interpretation für einen Sachverhalt ausgewählt wird, wird gleichzeitig unterstellt, dass der sprachliche Ausdruck Interpretationsbedingungen haben kann, die zum vorliegenden Sachverhalt passen. Aus meiner Sicht ist das der Hauptgrund dafür, warum die KomplementProposition typischerweise auf sprachlichen Ausdrücken basiert, deren Satisfaktionskriterien verhandlungsoffen sind, also im Rahmen einer direkt metasprachlichen Struktur fixiert werden könnten. Mit Reis (2013a: Anm. 26) und gegen v.a. Umbach (2016) glaube ich aber nicht, dass man diese Verwandtschaft zum metasprachlichen Gebrauch über eine entsprechende Selektionsbeschränkung für finden formulieren sollte. Fälle wie in (65) unten sprechen m.E. klar dafür, dass finden auch mit Propositionen kompatibel ist, bei denen es in keiner Weise um Satisfaktionskriterien der darin verwendeten sprachlichen Ausdrücke geht. 
Funktion: Der als-Satz begründet nicht, warum Ben groß ist, sondern warum man sagen kann, dass er groß ist; d.h., er liefert einen Grund dafür, warum der Sprecher die Interpretation ,Ben ist groß` für einen gegebenen Sachverhalt für gerechtfertigt hält.

(64) Ben ist in/SOfern groß, als er eins/NEUNzig groß ist $\backslash$.

Beachtenswert ist zudem als weitere Gemeinsamkeit, dass die Bezugsaussage Ben ist groß in beiden Fällen als Ganze ins Spiel kommt. So argumentiert Reis gegen die vor allem von Sæbø (2009) prominent gemachte Annahme, dass finden notwendig auf ein urteilersensitives Prädikat zielt; für finden mit finitem Komplement ist stattdessen entscheidend, dass die gewählte Proposition als Ganze eine mögliche Interpretation eines gegebenen Sachverhalts darstellt.

Trotz dieser suggestiven Parallelen unterscheiden sich aber finden und SIAGefüge in ihrer Distribution entscheidend. Für finden ist nach Reis (2013a) charakteristisch, dass es mit so genannten deskriptiven Interpretationsalternativen kompatibel ist, für die mit Fakten argumentiert werden kann, s. (65). Finden kann also die subjektive Einschätzung in vermeintlich faktbasierten Kontexten forcieren.

(65) a. [Kontext: Rätseln über den Ausgang des Tatorts]

Paul findet, dass der SCHWIEgersohn der Mörder ist. Schließlich hat er als einziger ein Motiv.

(Reis 2013a: [37a])

b. Tom findet, dass er $_{i}$ schwul ist, und das nur, weil er $r_{i}$ mit seinen grade mal 13 Jahren keine Freundin hat.

(Reis 2013a: [43’b])

c. [Kontext: Deutung eines Bildes, auf dem Peter halb angezogen vor demBett steht]

Paul findet, dass Peter ins Bett geht. Schließlich steht er halb angezogen vor dem Bett.

(Reis 2013a: [42b])

Entsprechende SIA-Gefüge sind hingegen klar abweichend, s. (66). Lizenziert werden sie nur bei Unterstützung durch ein explizites Modal oder ein explizites Einstellungsverb wie glauben (mit jeweils engem Skopus relativ zu insofern als), s. (67). 
(66) a. \# Der Mörder is in/SOfern der SCHWIEgersohn, als er der einzige mit Mo/TIV ist $\backslash$.

b. \# Tom $_{i}$ ist in/SOfern schwul, als er mit seinen grade mal 13 Jahren keine /FREUNdin hat .

c. \# Peter geht in/SOfern ins Bett, als er halbangezogen vor dem /BETT steht, er Richtung Bett /GUCKT und draußen die untergehende /SONne erkennbar ist $\backslash$.

(67) a. Der Mörder dürfte in/SOfern der SCHWIEgersohn sein, als er der einzige mit $M o / T I V$ ist $\backslash$.

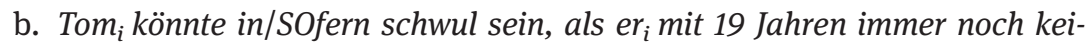
ne /FREUNdin hat .

c. In/SOfern, als er halbangezogen vor dem /BETT steht, er Richtung Bett /GUCKT und draußen die untergehende /SONne erkennbar ist, glaube ich, dass Peter ins BETT\geht.

Anders als finden forcieren also SIA-Gefüge keine subjektiv-epistemische Sachverhaltseinschätzung. Vielmehr liefern die jeweiligen als-Sätze Hinsichten, relativ zu denen der Sprecher einen Sachverhalt als objektiv gegeben präsentiert: Relativ zu den Fakten, die von den als-Sätzen in (66) und (67) beschrieben werden, kann jeweils der Sachverhalt, dass ein Sachverhalt möglich oder notwendig bzw. doxastisch zugänglich ist, als objektiv gültig gelten; deshalb sind die Beispiele in (67) akzeptabel. Die als-Sätze liefern hingegen keine guten Gründe dafür, warum die jeweils eingebetteten Sachverhalte selbst gültig sein sollten; deshalb sind die Beispiele in (66) abweichend. Ergänzt sei, dass sich vor diesem Hintergrund auch unmittelbar erklärt, warum SIA-Gefüge gut mit finden kombinierbar sind: Der als-Satz begründet jeweils, warum eine bestimmte subjektiv-epistemische Einstellung vorliegt, s. die Beispiele in (68).

(68) a. In/SOfern, als Martha einen /FEHler gemacht hat, finde ich, dass sie sich entSCHUL \digen sollte.

b. In/SOfern, als der Text die relevanten Punkte klar be/NENNT, finde ich ihn sehr geLUN\gen.

Diese Deutung der Distributionseigenschaften von SIA-Gefügen bestätigt sich mit Blick auf epistemisch-evidentiale Notwendigkeitsmodale. Entsprechende Beispiele sind mit finden inkompatibel, s. (69) sowie Reis (2013a: 415) zu möglichen Gründen für diese Inkompatibilität. Demgegenüber sind SIA-Gefüge hier möglich, s. (70). 
(69) a. ?? Er findet, dass die Zündler Kinder gewesen sein dürften. (Reis 2013a, [29b])

b. * Paul findet, dass Peter in Rom gewesen sein will. (Reis 2013a, [41a])

(70) a. Die Kinder dürften in/SOfern die Zündler gewesen sein, als man sie vom Tatort hat /WEGrennen sehen $\backslash$.

b. Peter will in/SOfern in Rom gewesen sein, als er tatsächlich glaubt, dass die Stadt mit der Theatinerkirche, also München, /ROM ist $\mid$.

Die Erklärung für diese Option liegt auf der Hand: Die epistemisch-evidentialen Notwendigkeitsmodale sind mit SIA-Gefügen deshalb kompatibel, weil der alsSatz jeweils eine Hinsicht liefert, relativ zu der die Annahme der spezifisch eingeschränkten Notwendigkeit objektiv rechtfertigbar ist.

Die zentrale Folgerung ist also, dass SIA-Gefüge keiner subjektiv-epistemischen Auswahl einer Sachverhaltsinterpretation dienen, sondern eine Sachverhaltsbeschreibung als relativ zu einer Tatsache objektiv gegeben präsentieren. Diese Deutung liefert gleichzeitig den Schlüssel dazu, warum SIA-Gefüge den Eindruck vermitteln, die Adäquatheit einer Sachverhaltsbeschreibung zu begründen: Die Relativierung auf eine Tatsache kann als dimensionale Spezifizierung von Sachverhaltsbeschreibungen verstanden werden; so wird die im alsSatz eingeführte Tatsache als konstitutiv für die Sachverhaltsbeschreibung auf der Matrixebene ausgewiesen. In diesem Sinne besteht der metasprachliche Effekt von SIA-Gefügen darin, Sachverhaltsbeschreibungen via Relativierung auf eine Tatsache auf eine dimensional spezifizierte Interpretation festzulegen. Bevor ich diese Überlegung im Rahmen der Formalisierung in Abschnitt 4 präzisieren werde, seien im nächsten Abschnitt weitere Evidenzen gegen die Annahme zusammengestellt, dass IA-Gefüge die Einführung einer subjektiv-epistemischen Einstellung involvieren.

\subsubsection{IA-Gefüge und die Einführung einer subjektiv-epistemischen Einstellung}

Reis (2013a) argumentiert dafür, dass finden - anders als von vor allem Sæbø (2009) angenommen - eine subjektiv-epistemische Einstellung einführt. Ein erstes zentrales Indiz liefern Fokussierungsdaten wie in (71). Das Beispiel drückt einen inhaltlichen Kontrast zwischen ,eine subjektive Meinung haben“ und ,wissen' aus, was sich nur so erklären lässt, dass finden selbst die entsprechende Einstellung einführt. 
(71) Paul FINdet nur, dass Tim ein Genie ist (er WEISS es nicht, denn seinen IQ gemessen hat er nie).

(Reis 2013a: [20])

LIA-Gefüge verhalten sich hier anders. Wie in Abschnitt 2.4 diskutiert erlauben sie die Fokussierung von Dimensionsalternativen, s. (72a). Es gibt aber keinen Hinweis auf eine zu finden analoge Fokussierbarkeit einer Einschätzungsrelation, s. (72b).

(72) a. Paul ist insofern ein Genie, als er immer gute IdEEN hat, nicht aber generell. Dafür hat er dann doch keinen hinreichend hohen IQ.

b. \# Paul ist inSOfern ein Genie, als er immer gute Ideen hat. Ich WEISS es aber nicht.

Weitere Testkriterien für die Existenz sprachlich eingeführter Entitäten liefern Umgebungen, die den expliziten Zugriff auf diese Entitäten voraussetzen. So zeigt das Beispiel in (73), dass finden eine subjektiv-epistemische Einstellung anaphorisch zugänglich macht; außerdem deutet das Beispiel in (74) darauf, dass Matrixprädikate auf die Einstellung selbst zugreifen können. Beide Befunde sprechen klar dafür, dass finden eine subjektiv-epistemische Einstellung denotiert. ${ }^{14}$

(73) Peter findet ${ }_{i}$ alles blöd $_{j}$ - Ja, das ${ }_{i / \# j}$ nervt.

(74) Ich finde blöd, dass Peter alles blöd findet.

$\approx$,Der Sprecher evaluiert das Urteil von Peter, dass alles blöd ist.“

₹ ,Der Sprecher evaluiert Zustände des Aus-der-Sicht-von-Peter-BlödSeins.'

Für IA-Gefüge hingegen fehlen entsprechende Evidenzen: Das anaphorische das in (75a) bzw. (75b) bezieht sich auf den spezifisch eingeschränkten Zuverlässigkeits-Zustand, nicht aber auf eine etwaig via insofern eingeführte Einstellung. So erklärt der Sprecher mit der Zustimmung in (75a) nicht für wahr, dass der Gesprächspartner oder eine andere Person eine bestimmte Einstellung zu Peters Zuverlässigkeit hat. Noch klarer tritt dieser Punkt bei Widerspruch wie in (75b) hervor: Ben bestreitet hier nicht, dass ein bestimmtes Urteil über Peters Zuverlässigkeit vorliegt. Unerheblich ist dabei jeweils, ob man das IA-Gefüge als LIA- oder als SIA-Gefüge liest.

14 Reis (2013a) diskutiert diese für Sæbøs (2009) Ansatz kritischen Aspekte nicht; sie unterstützen aber ihre Analyse. 


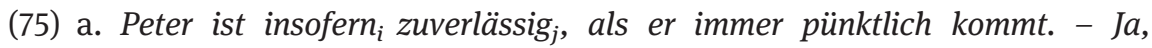
das $_{\# i / j}$ stimmt.

b. Peter ist insofern - und das bestreitet Ben - zuverlässig, als er immer pünktlich kommt.

₹ ,Peter ist insofern zuverlässig, als er immer pünktlich kommt, und Ben bestreitet, dass jemand entsprechend urteilt.'

₹ ,Peter ist insofern zuverlässig, als er immer pünktlich kommt, und Ben bestreitet, dass das stimmt.

Die folgenden Einbettungsstrukturen liefern ein analoges Bild (wobei allein LIA-Gefüge testbar sind, da nur diese Einbettung erlauben). So zeigt die Interpretation von (76), dass insofern keinen separaten Gegenstand für andere Matrixprädikate verfügbar macht. Zudem kann Matrixnegation nur bei finden, nicht aber bei insofern auf die Existenz einer subjektiv-epistemischen Einstellung zielen, s. den Kontrast in (77).

(76) Ich finde, dass Peter insofern zuverlässig ist, als er immer pünktlich kommt.

$\neq$,Sprecher findet, dass $x$ findet, dass Peter (in einer bestimmten Hinsicht) zuverlässig ist.

$\approx$,Sprecher findet, dass Peter (in einer bestimmten Hinsicht) zuverlässig ist.

(77) a. Es stimmt nicht, dass ich finde, dass sich Paul (in irgendeiner Hinsicht) blöd verhalten hat. Ich habe nämlich keine Meinung zu Pauls Verhalten.

b. \# Es stimmt nicht, dass Paul sich insofern blöd verhalten hat, als er Martha links liegen ließ. Ich habe nämlich keine Meinung zu Pauls Verhalten.

Die Befunde sprechen dafür, dass sich in IA-Gefügen Subjektivität zumindest nicht in Form einer direkt zugänglichen Einstellung manifestiert. $\mathrm{Zu}$ klären bleibt, ob es vielleicht andere Anzeichen einer $\mathrm{zu}$ finden vergleichbaren Form von Subjektivität gibt. Zum einen könnte in diesem Zusammenhang relevant sein, dass finden in notwendig nicht-subjektiven Kontexten abweichend ist, s. Reis (2013a: 393-394). So ist (78) deshalb markiert, weil man von Ärzten keine subjektiven Meinungen, sondern objektiv begründbare Einschätzungen erwartet. Zum anderen ist für finden charakteristisch, dass es gegen Widerspruch immunisiert, s. Umbach (2016) sowie Beispiel (79).

(78) A: Schwester, was sagt der Arzt?

a. \# Er findet, wir müssen Sie dabehalten.

b. \# Er findet Ihre Leberwerte zu hoch.

(Reis 2013a: [10]) 
(79) a. A: Ich finde Lakritze lecker.

b. \# B: Nein! Lakritze ist eklig.

(Umbach 2016: [4])

Doch auch diese Subjektivitätstests liefern für insofern negative Ergebnisse: So ist insofern in Fachdiskursen unauffällig, s. die SIA-Gefüge in (80). Auch erlaubt es Widerspruch, wie die LIA-Gefüge in (81) zeigen. Der Widerspruch kann sich dabei auf die Wahl der Dimension bzw. die restringierte Prädikation beziehen.

(80) A: Schwester, was sagt der Arzt?

a. Wir müssen sie in/SOfern dabehalten, als noch einige Unter/SUchungen anstehen \.

b. Ihre Leberwerte sind in/SOfern zu hoch, als bestimmte Grenzwerte für /LEberenzyme überschritten werden\.

(81) A: Ben war in/SOfern ein Idiot, als er Martha einen KOCH\topf geschenkt hat.

a. B: Nein, er war in/SOfern ein Idiot, als er Paul RO\sen geschenkt hat.

b. B: Nein, das war er nicht - Martha wollte unbedingt einen Kochtopf.

Festhalten lässt sich also, dass insofern keine subjektiv-epistemische Einstellung einführt und dass auch andere Indizien für eine spezifisch subjektive Bedeutung fehlen. Dieses Ergebnis unterstreicht, dass es in IA-Gefügen nicht um die subjektive Auswahl von Interpretationen geht, sondern um als objektiv präsentierte dimensional spezifizierte lexikalische Prädikationen bzw. Sachverhaltsbeschreibungen.

\subsection{Zwischenfazit}

LIA-Gefüge setzen die Kombination mit einer lexikalisch angelegten mehrdimensionalen Prädikation im Bezugssatz voraus, sind aber nicht sensitiv bezüglich urteilerabhängiger Prädikate. Ihr metasprachlicher Effekt besteht darin, dimensional spezifizierte Interpretationen mehrdimensionaler lexikalischer Prädikationen zu bestimmen. Sie dienen hingegen nicht dazu, ein Prädikat zu disambiguieren oder für seine Interpretation Satisfaktionskriterien festzulegen.

Für SIA-Gefüge spielt lexikalisch angelegte Mehrdimensionalität keine Rolle; relevant ist stattdessen, ob der als-Satz eine Dimension verfügbar macht, relativ zu der der vom Bezugssatz beschriebene Sachverhalt als objektiv gegeben gelten kann. SIA-Gefüge können deshalb keine subjektiven Einschätzungen 
forcieren, sind aber verträglich mit expliziten Modalen (inklusive epistemischevidentialen Notwendigkeitsmodalen) und Einstellungsbeschreibungen. Ihr metasprachlicher Effekt besteht nicht darin, wie finden Interpretationen von Sachverhalten auszuwählen, sondern darin, Sachverhaltsbeschreibungen über die via als-Satz eingeführte Hinsicht dimensional zu spezifizieren. Die Intuition, der als-Satz „begründe“ die Interpretation eines Sachverhalts, ergibt sich entsprechend daraus, dass der als-Satz die konstitutive Dimension der dimensional spezifizierten Sachverhaltsbeschreibung beisteuert.

Dass IA-Gefüge keine spezifische subjektive Bedeutung involvieren, wurde abschließend anhand weiterer Subjektivitätstests bestätigt: Weder erlauben IAGefüge die Fokussierung einer subjektiv-epistemischen Einstellung noch den sprachlich expliziten Bezug auf eine solche; sie beschränken nicht auf Subjektivität lizenzierende Diskurse und immunisieren nicht gegen Widerspruch.

\section{Zur Ausbuchstabierung der semantischen Komposition}

\subsection{Vorbemerkungen}

Aus den ermittelten Eigenschaften der beiden IA-Gefügevarianten ergibt sich als gemeinsamer semantischer Kern, dass der als-Satz einen Anker für eine dimensionale Einschränkung einer mehrdimensionalen Bezugsgröße liefert: In LIA-Gefügen zielt die Einschränkung auf die Mehrdimensionalität einer lexikalischen Prädikation; in SIA-Gefügen zielt die Einschränkung auf die Mehrdimensionalität einer sentential gegebenen Sachverhaltsbeschreibung. In den folgenden Abschnitten möchte ich diese These semantisch präzisieren. Dazu muss zunächst geklärt werden, wie Hinsichten und die darauf gründenden dimensionalen Interpretationen repräsentiert und kompositional integriert werden können. Ich werde meinen Vorschlag auf der Analyse von with respect to q-Phrasen in Sassoon (2013a) und Sassoon (2013b) aufbauen. Sassoon (2013a: 337-338) schlägt vor, dass diese ein mehrdimensionales Prädikat modifizieren und dabei durch die Setzung einer Dimension in ein dimensional spezifiziertes Prädikat überführen, s. auf das Deutsche übertragen z. B. (82). ${ }^{15}$

15 Sassoon (2013a) gibt als Alternative an, mehrdimensionale Prädikate von vornherein mit einem optionalen Hinsichts-Argument auszustatten, das durch die with respect to $q$-Phrasen gesättigt wird. Ich halte es für sparsamer, die Einführung einer Dimension an die entsprechenden Phrasen selbst zu knüpfen und folge deshalb hier der Modifikator-Analyse. Erwähnt sei in diesem Zusammenhang noch zweierlei. Erstens: Sassoon relativiert die Interpretation dimensional spezifizierter Prädikationen zusätzlich auf Kontextindizes; diese spielen in meiner 
(82) $\llbracket$ attraktiv in Hinsicht auf $q \rrbracket$

$=\lambda x \cdot$ in-Hinsicht-auf' $\left(x, \lambda y \cdot \operatorname{attraktiv}^{\prime}(y), q\right)$

$=\lambda x \cdot$ in-Hinsicht-auf $\left(x, \operatorname{attraktiv}^{\prime}, q\right)$

Entsprechend verallgemeinert verstehe ich dim' als eine Funktion, die beliebige Prädikationen $P$ auf die Menge ihrer dimensional spezifizierten Interpretationen abbildet, s. (83a) (wobei der Vektor anzeigt, dass die Prädikation eine variable Menge an Argumentstellen aufweisen kann). Demgegenüber kann Hinsicht' als Funktion definiert werden, die Mengen von dimensional spezifizierten Interpretationen von Prädikationen auf die Menge der jeweiligen Dimensionen bzw. Hinsichten abbildet, s. (83b). ${ }^{16}$

(83) a. $\operatorname{dim}^{\prime}(P)=\left\{\lambda \vec{x}\right.$.in-Hinsicht-auf' $\left(\vec{x}, \lambda \vec{y} \cdot P(\vec{y}), q_{1}\right)$, $\lambda \vec{x}$.in-Hinsicht-auf' $\left.\left(\vec{x}, \lambda \vec{y} \cdot P(\vec{y}), q_{2}\right), \ldots\right\}$

b. $\operatorname{Hinsicht}^{\prime}\left(\operatorname{dim}^{\prime}(P)\right)=\left\{q_{1}, q_{2}, \ldots\right\}$

Diese Verallgemeinerung lässt den ontologischen Status von Dimensionen bzw. Hinsichten bewusst offen und ermöglicht so, dass diese relativ zur Art des jeweiligen Prädikats $P$ bestimmt werden können. Für die von Sassoon (2013a) behandelten deskriptiven lexikalischen Prädikate ist charakteristisch, dass ihre Hinsichten selbst wieder inhaltlich-deskriptiver Natur sind; vgl. z. B. Kosten (etw. ist attraktiv in Hinsicht auf seine Kosten), Zeit (etw. ist attraktiv in Hinsicht auf die Zeit, die es beansprucht), Gesundheit (jm. geht es gut in Hinsicht auf seine Gesundheit), Farben (etw. ist gelungen in Hinsicht auf seine Farbe), Größen (etw. ist identisch in Hinsicht auf die jeweilige Größe) etc. Damit dienen Hinsichten dazu, den Gültigkeitsbereich von Prädikaten inhaltlich-deskriptiv explizit einzugrenzen und so in spezifischere Prädikate zu überführen. Da dieser intuitive Zugriff für das Hauptanliegen des vorliegenden Artikels ausreicht, möchte

\footnotetext{
Analyse keine zentrale Rolle und werden deshalb im Folgenden ausgelassen. Zweitens: Da es mir hauptsächlich um die Frage geht, wie insofern als zur expliziten Dimensionseinschränkung beiträgt, möchte ich mich agnostisch bezüglich der Frage verhalten, wie Dimensionen dann bestimmt werden, wenn eine solche explizite Einschränkung fehlt. Sassoon (2013a) rückt genau diese Frage in den Vordergrund. Sie argumentiert dafür, dass Adjektive unterschiedliche Defaults bezüglich der Bindung von Dimensionen aufweisen; so befördert z. B. krank eine Existenzquantifikation, gesund hingegen eine Allquantifikation (vgl. Ben ist krank. $\approx$,Ben ist in einer Hinsicht krank.' vs. Ben ist gesund. $\approx$,Ben ist in allen Hinsichten gesund.`).

16 Ich trenne damit begrifflich zwischen dimensionalen Interpretationen von Prädikationen und den Dimensionen bzw. Hinsichten selbst. Bei Sassoon (2013a) ist das m. E. nicht ganz klar; so bezeichnet sie die dimensional spezifizierten Prädikate selbst, also Elemente von dim' $(P)$, als Dimensionen.
} 
ich auf eine präzise ontologische Charakterisierung verzichten; erwähnt sei lediglich, dass es dafür verschiedene plausible Anschlussoptionen gibt. Beispielsweise lassen sich Hinsichten als generalisierte Maßfunktionen und damit als Abbildungen von Individuen in Werte in Attributsräumen analysieren; so bilden Kosten ein Individuum auf einen bestimmten Geldbetrag ab, Farben bilden auf einen Farbwert ab, Größe auf einen Größegrad etc. (s. zu Maßfunktionen in der Gradsemantik Kennedy [1999], Beck [2011] und zum generalisierten Pendant Umbach und Gust [2014], Umbach [2016]). Eine alternative Möglichkeit besteht darin, Hinsichten als partikularisierte Eigenschaften zu analysieren; entsprechend handelt es sich bei den Kosten von etwas, der Farbe von etwas, der Größe von jemandem etc. um konkrete Entitäten in der Welt, die sich in ihren Trägern manifestieren (s. Moltmann 2009, Moltmann 2013). Wesentlich für die Analyse von IA-Gefügen wird hingegen sein, dass nur die Interpretation von LIA-Gefügen auf inhaltlich-deskriptiven Hinsichten beruht, die von SIA-Gefügen hingegen den Rückgriff auf eine andere Art Hinsicht erfordert; s. dazu die Argumentation in Abschnitt 4.3.

Die zweite Festlegung betrifft die Repräsentation von Sätzen bzw. Propositionen. Hierzu werde ich Situationssemantik im Sinne von Kratzer (2002, 2010) nutzen. Demnach sind Situationen basale Entitäten, die Teil-Ganzes-Beziehungen eingehen können, s. die Bezeichnung $s^{\prime} \leq s$ für,$s^{\prime}$ ist Teil von $s^{\prime}$. Entsprechend können Situationen unterschiedlich groß sein. Situationen, die keine Teile von anderen Situationen bilden und damit maximal sind, entsprechen Welten. Für die Analyse wesentlich werden vor allem zwei Situationstypen, die unterschiedlich große Weltausschnitte bezeichnen: Topiksituationen sowie Fakten. Unter Topiksituationen verstehe ich grob die partikulare Situation, über die ein Satz eine Aussage macht (s. zu diesem an John L. Austin orientierten Situationsbegriff Kratzer [2010: Kap. 3]; ähnliche Konzeptionen finden sich in Klein [1994] oder Maienborn [2003a]). Konkret nehme ich an, dass die mit Sätzen einhergehenden Sachverhaltsbeschreibungen Prädikationen über Topiksituationen sind, s. (84) mit $s^{*}$ für Topiksituationen und $s$ für den darin enthaltenen Attraktivitäts-Zustand.

$$
\text { [Die Lösung ist attraktiv } \rrbracket=\lambda s^{*} \exists s\left[s \leq s^{*} \wedge \text { attraktiv'( }\left(l\left[\operatorname{Lösung}^{\prime}(l)\right]\right)(s)\right]
$$

Fakten sind demgegenüber spezifischere Entitäten, die als Propositionen exemplifizierende Situationen definiert werden können, s. dazu (85) im Anschluss an Kratzer (2002: Def. 6).

(85) Eine Situation $s$ ist ein Fakt $f$, das eine Proposition $p$ exemplifiziert, gdw. es für alle $s^{\prime}$, sodass $s^{\prime} \leq s$ und $s^{\prime} \notin / p$, eine Situation $s^{\prime \prime}$ gibt, sodass gilt: 
(i) $s^{\prime} \leq s^{\prime \prime} \leq s$

(ii) $s^{\prime \prime}$ ist eine minimale Situation, in der $p$ wahr ist (d.h., es gibt keine echten Teile von $s^{\prime \prime}$, in denen $p$ wahr ist).

Kratzer illustriert die Definition u. a. an unterschiedlichen Teekannen-Szenarien. So ist z. B. eine Situation $s$, in der sich drei Teekannen befinden und sonst nichts, ein Fakt, das die Proposition $p$ Es gibt drei Teekannen exemplifiziert: Situationen $s^{\prime}$, in denen $p$ nicht gilt (etwa eine Situation mit nur zwei Teekannen), lassen sich zu einer minimalen Situation $s^{\prime \prime}$ erweitern, in der $p$ wahr ist (nämlich der Situation mit drei Teekannen). Demgegenüber ist eine Situation $s$, in der sich drei Teekannen und eine Schere befinden und sonst nichts, kein Fakt, das die Proposition $p$ exemplifiziert: Die Situation $s^{\prime}$, in der sich nur die Schere befindet, ist ein Teil von $s$ und erfüllt $p$ nicht; sie lässt sich aber nicht zu einer minimalen Situation $s^{\prime \prime}$ erweitern, in der $p$ gilt, da ja immer die Schere im Spiel bleibt.

Im Folgenden möchte ich zeigen, wie die genannten Komponenten zusammengeführt und produktiv zur Analyse dimensionaler Spezifizierungen via IAGefüge genutzt werden können.

\subsection{Zur Interpretation von LIA-Gefügen}

Die bisherige Untersuchung hat gezeigt, dass LIA-Gefüge der dimensionalen Einschränkung einer lexikalischen, also inhaltlich bestimmten deskriptiven Prädikation dienen, s. wiederholt (86).

(86) a. Die Lösung ist in/SOfern attraktiv, als sie GÜN\stig ist.

b. ,Die Lösung ist in einer Hinsicht attraktiv, die sich daraus ergibt, dass sie günstig ist.‘

Damit ist das Ziel der dimensionalen Spezifizierung völlig analog zu den von Sassoon (2013a) beschriebenen Fällen; der Unterschied besteht darin, dass die Dimension selbst nicht direkt über eine PP ins Spiel kommt, sondern aus dem als-Satz erschlossen werden muss. Zur Ausbuchstabierung der Komposition schlage ich für insofern als folgende Bedeutung vor: ${ }^{17}$

17 Der Eintrag wirft die Frage auf, ob man die Bedeutung von insofern als dekomponieren kann. Eine naheliegende Option besteht darin, die Komponente als an die Einführung des Fakts, so an dessen anaphorische Aufnahme, fern an die Hinsicht und in an die Mittlerrolle von Hinsicht und Matrixprädikation zu knüpfen. In diesem Zusammenhang wäre dann auch zu klären, wie sich insofern als zu den in der Einleitung erwähnten Strukturalternativen mit dass bzw. insoweit verhält. Ich möchte diese Frage hier aber offen lassen und behandle deshalb insofern als als konstruktionales Ganzes. 


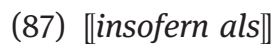

$=\lambda p \lambda P:\left|\operatorname{dim}^{\prime}(P)\right|>1 \lambda \vec{x} . \exists f \in \operatorname{exempl}^{\prime}(p) \exists q \in \operatorname{Hinsicht}^{\prime}\left(\operatorname{dim}^{\prime}(P)\right)$

[in-Hinsicht-auf' $\left(\vec{x}, P, q^{f}\right)$,

wobei $q^{f}=q$, das über das Fakt $f$ zu erschließen ist]

Gemäß (87) bildet insofern als die als-Satz-Proposition $p$ auf ein $p$ exemplifizierendes Fakt $f$ sowie die Matrixprädikation $P$ auf eine dimensional spezifizierte Interpretation ab; dabei ist dessen Dimension $q$ relativ $\mathrm{zu} f \mathrm{zu}$ bestimmen. Die an $P$ geknüpfte Information $\left|\operatorname{dim}^{\prime}(P)\right|>1$ erfasst, dass insofern als die Applikation auf eine mehrdimensionale Prädikation präsupponiert (die Kardinalität der Menge $\operatorname{dim}^{\prime}(P)$ muss größer als 1 sein).

Für Beispiel (86) ergibt sich damit die folgende Derivation. Aus der Anwendung auf die als-Satz-Proposition resultiert (88). Da LIA-Gefüge in ihren Matrixsatz strukturell integriert sind, gilt die sich anschließende Verrechnung dem vom Matrixsatz gelieferten lexikalischen Material, in diesem Fall also der von attraktiv beigesteuerten mehrdimensionalen Prädikation in (89). Daraus folgt deren Überführung in die dimensional spezifizierte Prädikation in (90). Die Integration des Subjektsarguments sowie Situationsbindung ergeben schließlich die finale Repräsentation in (91). (Da Topiksituationen für den spezifischen Beitrag von LIA-Gefügen keine Rolle spielen, bleiben diese hier unberücksichtigt.)

(88) [insofern als sie günstig ist

$=\lambda P:\left|\operatorname{dim}^{\prime}(P)\right|>1 \lambda \vec{x} . \exists f \in \operatorname{exempl}^{\prime}\left(\lambda s^{\prime}\right.$.günstig' $\left.\left(l l\left[\operatorname{Lösung}^{\prime}(l)\right]\right)\left(s^{\prime}\right)\right)$

$\exists q \in \operatorname{Hinsicht}^{\prime}\left(\operatorname{dim}^{\prime}(P)\right)\left[\right.$ in-Hinsicht-auf' $\left(\vec{x}, P, q^{f}\right)$,

wobei $q^{f}=q$, das über das Fakt $f$ zu erschließen ist]

(89) $\llbracket$ attraktiv

$=\lambda x^{\prime} \lambda s^{\prime \prime \prime}$.attraktiv' $\left(x^{\prime}\right)\left(s^{\prime \prime \prime}\right)$

(90) [insofern attraktiv als sie günstig ist]

$=\lambda x \lambda s . \exists f \in \operatorname{exempl}^{\prime}\left(\lambda s^{\prime}\right.$.günstig' $\left.\left(\iota l\left[\operatorname{Lösung}^{\prime}(l)\right]\right)\left(s^{\prime}\right)\right)$

$\exists q \in \operatorname{Hinsicht}^{\prime}\left(\operatorname{dim}^{\prime}\left(\lambda x^{\prime} \lambda s^{\prime \prime \prime}\right.\right.$.attraktiv' $\left.\left.\left(x^{\prime}\right)\left(s^{\prime \prime \prime}\right)\right)\right)$

[in-Hinsicht-auf' $\left(s, x, \lambda x^{\prime} \lambda s^{\prime \prime \prime}\right.$.attraktiv' $\left.\left(x^{\prime}\right)\left(s^{\prime \prime \prime}\right), q^{f}\right)$, wobei $q^{f}=q$, das über das Fakt $f$ zu erschließen ist]

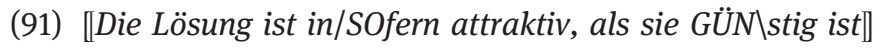

$=1$ gdw. $\exists s \exists f \in \operatorname{exempl}^{\prime}\left(\lambda s^{\prime}\right.$.günstig' $\left(l\left[\left[\operatorname{Lösung}^{\prime}(l)\right]\right)\left(s^{\prime}\right)\right)$

$\exists q \in \operatorname{Hinsicht}^{\prime}\left(\operatorname{dim}^{\prime}\left(\lambda x^{\prime} \lambda s^{\prime \prime \prime}\right.\right.$.attraktiv' $\left.\left.\left(x^{\prime}\right)\left(s^{\prime \prime \prime}\right)\right)\right)$

[in-Hinsicht-auf'(s,ll[Lösung $(l)], \lambda x^{\prime} \lambda s^{\prime \prime \prime}$.attraktiv' $\left.\left(x^{\prime}\right)\left(s^{\prime \prime \prime}\right), q^{f}\right)$, wobei $q^{f}=q$, das über das Fakt $f$ zu erschließen ist] 
$=1 \mathrm{gdw} . \exists s \exists f \in \operatorname{exempl}^{\prime}\left(\lambda s^{\prime}\right.$.günstig' $\left.\left(l l\left[\operatorname{Lösung}^{\prime}(l)\right]\right)\left(s^{\prime}\right)\right)$

$\exists q \in \operatorname{Hinsicht}^{\prime}\left(\operatorname{dim}^{\prime}\left(\operatorname{attraktiv}^{\prime}\right)\right)$

[in-Hinsicht-auf'(s,ll[Lösung' $(l)]$, attraktiv $\left.^{\prime}, q^{f}\right)$, wobei $q^{f}=q$, das über das Fakt $f$ zu erschließen ist]

In Prosa: Der Satz (86a) ist wahr gdw. es eine Situation $s$ des In-Hinsicht-auf$\mathrm{q}^{\mathrm{f}}$-Attraktiv-Seins der Lösung gibt, wobei $q^{f}$ eine Attraktivitäts-Hinsicht ist, die sich aus der Tatsache ergibt, dass die Lösung günstig ist. Welche Vorzüge vereint der Vorschlag?

Erstens wird die unabhängig motivierte strukturelle Integration von IA-Gefügen für die Bedeutungsherleitung systematisch nutzbar gemacht. So sorgt nicht die spezifische Bedeutung von insofern als, sondern ihre kompositional vermittelte Bezugsgröße dafür, dass die dimensionale Einschränkung hier lexikalischen deskriptiven Prädikationen wie attraktiv gilt. Dies ist die Grundlage dafür, dass der Eintrag in (87) unverändert für SIA-Gefüge übernommen werden kann, s. dazu die spätere Analyse in Abschnitt4.3.

Zweitens erfasst die Analyse unmittelbar, warum LIA-Gefüge keine eindimensionalen Matrixprädikate wie schwer erlauben. Diese erfüllen die an $P$ gestellte Mehrdimensionalitäts-Präsupposition nicht, s. $\mid \operatorname{dim}^{\prime}\left(\right.$ schwer' $\left.^{\prime}\right) \mid=1$.

Drittens liefert das Ergebnis der Komposition eine plausible Basis für eine pragmatisch gesteuerte Anreicherung der Dimensionsvariablen $q$. Dazu möchte ich die wesentlichen Eckpunkte der Spezifizierung grob skizzieren. Auf der Basis der semantischen Form in (91) stellt sich die Frage, was eine möglichst plausible, um eine Hinsicht angereicherte Interpretation der Attraktivitätsprädikation ist, sodass sich die relevante Hinsicht auf die Tatsache, dass die Lösung günstig ist, bezieht. Schematisch kann man das als Suchbefehl nach einem möglichst plausiblen Kandidaten für eine angereicherte konzeptuelle Struktur A verstehen, die folgenden abduktiven Schluss rechtfertigt (s. allgemeiner zu Abduktion Hobbs et al. [1993]; das folgende Schema folgt Maienborn [2003b: 491]).

(92) A $\rightarrow$ B (konzeptuelles Wissen)

B (unterspezifizierte semantische Form)

A (angereicherte konzeptuelle Struktur)

Was sind nun Kandidaten im Fall von (91)? - Zunächst lässt sich festhalten, dass in LIA-Gefügen die einfachste aller Spezifizierungen, nämlich eine Identifikation der Hinsicht $q$ mit dem Fakt $f$ selbst, aus ontologischer Perspektive ausgeschlossen ist. Dies liegt daran, dass es hier um Hinsichten deskriptiver lexika- 
lischer Prädikate geht und dafür Fakten keinen adäquaten sortalen Typ liefern. Stattdessen müssen, wie in Abschnitt 4.1 motiviert, entsprechende Hinsichten ebenfalls inhaltlich deskriptiver Art sein. Im vorliegenden Fall ist die Instantiierung von $q$ als Kosten-Hinsicht plausibel. Dies ist sowohl eine mögliche Hinsicht von Attraktivität als auch unmittelbar auf die Tatsache bezogen, dass die Lösung günstig ist; erfassbar ist dies über ein Axiom wie (93). ${ }^{18}$

(93) $\forall q f y$.

$\operatorname{Hinsicht}^{\prime}(q) \wedge$ erschlossen-über' $(q, f) \wedge$ günstig' $(y)(f) \wedge$ etc $^{\prime}$ Kosten

$\rightarrow \operatorname{Kosten}^{\prime}(q, y)$

,Wenn etwas eine Hinsicht ist, die über eine Tatsache, dass y günstig ist, erschlossen wird, dann kann es sich um eine Kosten-von- $y$-Hinsicht handeln.

Demgegenüber würde z. B. die Instantiierung von $q$ als Zeit-Hinsicht scheitern. Zwar kann etwas zeitlich attraktiv sein, doch hätte die Zeit-Hinsicht nichts unmittelbar mit der Tatsache zu tun, dass die Lösung günstig ist; so ist der Schluss in (94) konzeptuell abweichend.

(94) \# $\forall q f y$.

$\operatorname{Hinsicht}^{\prime}(q) \wedge$ erschlossen-über' $(q, f) \wedge$ günstig' $(y)(f) \wedge \operatorname{etc}^{\prime}{ }_{\text {Zeit }} \rightarrow \operatorname{Zeit}^{\prime}(q, y)$ ,Wenn etwas eine Hinsicht ist, die über eine Tatsache, dass y günstig ist, erschlossen wird, dann kann es sich um eine Zeit-von-y-Hinsicht handeln.

Man mag sich fragen, warum die Spezifikation von $q$ als Kosten-Hinsicht im vorliegenden Beispiel zwingend ist. Der offenkundige Grund dafür liegt in der Spezifizität der vom als-Satz beigesteuerten Tatsache, s. das explizit auf Kosten zielende Adjektiv günstig. Dies bestätigt die Annahme, dass die zentrale Funktion des als-Satzes darin besteht, eine dimensionale Einschränkung der Matrixprädikation durch eine relativ dazu feinkörnige Information zu rechtfertigen. Weitere Bestätigung ergibt sich aus der Beobachtung, dass zwischen Matrixprä-

18 Die Formulierung entsprechender Axiome nutzt so genannte etc'-Prädikate, s. dazu grundsätzlich Hobbs et al. (1993: 84-87). Diese sind nötig, um den für Abduktion charakteristischen Schluss von einer Unter- auf eine Obermenge zu repräsentieren. Sie sind Platzhalter für all jene (nicht explizit auszubuchstabierenden) Eigenschaften, die einen entsprechend ungültigen Schluss in ein wohlgeformtes Muster überführen, s. (ia) vs. (ib).

(i) a. ${ }^{\star} \forall x \cdot \operatorname{Baum}^{\prime}(x) \rightarrow \operatorname{Buche}^{\prime}(x)$

b. $\forall x \cdot \operatorname{Baum}^{\prime}(x) \wedge \operatorname{etc}^{\prime}{ }_{\text {Buche }}(x) \rightarrow \operatorname{Buche}^{\prime}(x)$ 
dikation und als-Satz eine Asymmetrie besteht, s. (95): Liefert der als-Satz eine relativ zur Matrixprädikation weniger spezifische Tatsache, kann diese nicht als adäquater Anker für eine dimensionale Einschränkung der Matrixprädikation dienen.

(95) a. \# Die Lösung ist in/SOfern günstig, als sie attrakTIV ist.

b. \# Ben ist in/SOfern gesund, als es ihm GUT\ geht.

c. \# Die Blumen passen in/SOfern farblich zueinander, als sie SCHÖN\sind.

Nicht zuletzt sagt die Analyse voraus, dass man die vom als-Satz benannte Tatsache in ihrer Feinkörnigkeit angemessen ernst nehmen muss. Aufschlussreich ist in diesem Zusammenhang der folgende Kontrast:

(96) a. Ben geht es in/SOfern gut, als er geSUND $\backslash$ ist.

b. \# Ben geht es in/SOfern gut, als es um GeSUND $\backslash$ heit geht.

Die als-Sätze in (96a) sowie (96b) rekurrieren beide auf Gesundheit und damit auf eine grundsätzlich plausible Dimension für die Beschränkung der Matrixprädikation gut geh-. Dies allein ist aber noch kein hinreichender Lizenzierungsfaktor: Der Bezug auf den spezifischen Gesundheitszustand von Ben ermöglicht ein entsprechendes LIA-Gefüge, s. (96a). Pragmatisch abweichend ist hingegen (96b): Die Tatsache, dass es um Gesundheit geht, ist (im Normalfall) kein passender Anker für die Frage, in welcher Hinsicht man über jemanden prädizieren kann, dass es ihm gut geht. Interpretierbar ist ein solcher Satz nur dann, wenn man einen entsprechenden Bezug kontextuell forciert, s. dazu (97).

(97) [Kontext: Party]

A: Wie geht's denn unserem Gesundheitsfanatiker in der Küche? - B: Ach, dem geht's nicht nur insofern gut, als es um Gesundheit geht, sondern auch insofern, als seine Angebetete ihm dabei an den Lippen hängt.

Die von den als-Sätzen eingeführten Tatsachen sind also in ihrer spezifischen Informativität für den Anreicherungsprozess relevant; gleichzeitig besteht aber ein kontextuell bestimmtes Anpassungspotenzial. Damit ist außerdem ein Grund dafür benannt, warum Sprecher die mit LIA-Gefügen verknüpfte indirekte Strategie einer dimensionalen Spezifizierung überhaupt nutzen: LIA-Gefüge ermöglichen es, potenziell sehr feinkörnige und eigenwillige Tatsachen als Anker für dimensionale Spezifizierungen ins Spiel zu bringen. 


\subsection{Zur Interpretation von SIA-Gefügen}

In SIA-Gefügen gilt die dimensionale Beschränkung Sätzen bzw. Sachverhaltsbeschreibungen als Ganzen, s. wiederholt (98).

(98) a. Die Lösung ist in/SOfern attraktiv, als sie /GÜNstig ist .

b. ,In Hinsicht darauf, dass die Lösung günstig ist, kann man sagen, dass die Lösung attraktiv ist.‘

Meines Wissens ist in der Literatur bisher nicht explizit diskutiert worden, was man sich in einem solchen Fall unter einer Dimension bzw. einer dimensionalen Spezifizierung überhaupt vorstellen soll. ${ }^{19}$ Mein Vorschlag basiert auf der unabhängig motivierten Annahme (s. die einleitenden Bemerkungen in Abschnitt 4.1), dass Sachverhaltsbeschreibungen als Prädikationen über Topiksituationen verstanden werden können. Mit dieser Annahme geht einher, dass SIA-Gefüge eine funktionale Bezugsgröße haben. So sind Topiksituationen nicht unmittelbar durch inhaltlich-deskriptive Informationen gekennzeichnet, sondern durch das, was in ihnen der Fall ist, m.a. W., durch ihre via s-Relation verknüpften Subsituationen. ${ }^{20}$ Dieser Hintergrund motiviert folgende Überlegung: Dimensionen von topiksituationalen Prädikationen sind die in Topiksituationen gültigen Tatsachen selbst; entsprechend lassen sich dimensionale Spezifizierungen von topiksituationalen Prädikaten als jene Prädikationen verstehen, die ein spezifischeres Bild der gegebenen Tatsachen liefern. Das heißt, dass die dimensionale Spezifizierung einer topiksituationalen Prädikation A einer topiksituationalen Prädikation B entspricht, die sich durch das Gegebensein

19 Die Analyse in Sassoon (2013a) und Sassoon (2013b) gilt deskriptiven Prädikaten, s. oben. Bartsch (1986) unterscheidet zwischen $\mathrm{u}$. a. adsententialen dimensionalen Spezifizierern wie (ia) and adverbialen Fällen wie (ib).

(i) a. With respect to health, John is fine.

b. John is fine healthwise.

(Bartsch 1986: 303)

Im Ansatz entspricht das der auch hier verteidigten Perspektive, dass die dimensionale Spezifizierung unterschiedliche Zielgrößen haben kann. Allerdings ist der Standpunkt weder syntaktisch ausgearbeitet noch wird er semantisch ausbuchstabiert. Bartsch konstatiert zwar, dass sich beide Fälle typenlogisch unterscheiden würden, macht aber m. E. in keiner Weise transparent, welche interpretatorisch greifbaren Konsequenzen die Differenz hat.

20 Natürlich wird das, was der Fall ist, durch inhaltlich-deskriptive Relationen ausgedrückt. Das heißt aber gerade nicht, dass Topiksituationen aus diesen inhaltlich-deskriptiven Relationen als solchen bestehen. Vielmehr bestehen sie aus den Gegebenheiten, die von diesen Relationen nur beschrieben werden. 
einer gegenüber A spezifischeren Tatsache auszeichnet. Dies passt ganz unmittelbar zur Interpretation von SIA-Gefügen wie in (98): Relativ zur Tatsache, dass die Lösung günstig ist, wird über eine gegebene Topiksituation die Aussage gemacht, dass die Lösung attraktiv ist. Intuitiv ist dabei die vom als-Satz eingeführte Tatsache eine gegenüber der Matrixprädikation spezifischere Information; gleichzeitig rechtfertigt diese Tatsache, warum man die Matrixprädikation überhaupt als gültig assertieren kann (was der für SIA-Gefüge diagnostizierten Begründungsintuition entspricht). Im Folgenden möchte ich die formale Implementierung der skizzierten Idee vorstellen und ihre Konsequenzen diskutieren.

Zunächst zur semantischen Komposition: Die Verrechnung des als-Satzes ist für SIA- und LIA-Gefüge identisch, s. wiederholt (99) bzw. (100) (= [87] und [88] oben). Der fehlende strukturelle Unterschied zwischen den Gefügen auf dieser Ebene sagt eine solche Uniformität regulär voraus.

$$
\begin{aligned}
& \text { 【insofern als } \rrbracket \\
& =\lambda p \lambda P:\left|\operatorname{dim}^{\prime}(P)\right|>1 \lambda \vec{x} \cdot \exists f \in \operatorname{exempl}^{\prime}(p) \exists q \in \operatorname{Hinsicht}^{\prime}\left(\operatorname{dim}^{\prime}(P)\right) \\
& \quad\left[\text { in-Hinsicht-auf' }\left(\vec{x}, P, q^{f}\right),\right. \\
& \quad \text { wobei } q^{f}=q \text {, das über das Fakt } f \text { zu erschließen ist] }
\end{aligned}
$$

(100) 【insofern als sie günstig ist]

$=\lambda P:\left|\operatorname{dim}^{\prime}(P)\right|>1 \lambda \vec{x} . \exists f \in \operatorname{exempl}^{\prime}\left(\lambda s^{\prime}\right.$.günstig' $\left.\left(l l\left[\operatorname{Lösung}^{\prime}(l)\right]\right)\left(s^{\prime}\right)\right)$

$\exists q \in \operatorname{Hinsicht}^{\prime}\left(\operatorname{dim}^{\prime}(P)\right)\left[\right.$ in-Hinsicht-auf' $\left(\vec{x}, P, q^{f}\right)$,

wobei $q^{f}=q$, das über das Fakt $f$ zu erschließen ist]

Der entscheidende Unterschied ergibt sich hingegen aus der strukturellen Desintegration von SIA-Gefügen. Anders als in LIA-Gefügen ist damit der Zugriff auf eine lexikalisch verankerte Binnenkomponente des Matrixsatzes versperrt; vorausgesagt wird stattdessen die Verrechnung mit der Sachverhaltsbeschreibung als Ganzer, d.h. der vom Matrixsatz denotierten topiksituationalen Prädikation in (101) (mit $s^{+}$für Topiksituation). Dies ergibt (102).

(101) «Die Lösung ist attraktiv》

$=\lambda s^{+} . \exists s\left[s \leq s^{+} \wedge \operatorname{attraktiv}^{\prime}\left(\operatorname{ll}\left[\operatorname{Lösung}^{\prime}(l)\right]\right)(s)\right]$

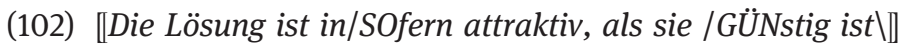

$=\lambda s^{*} . \exists f \in \operatorname{exempl}^{\prime}\left(\lambda s^{\prime}\right.$.günstig' $\left.\left(\iota l\left[\operatorname{Lösung}^{\prime}(l)\right]\right)\left(s^{\prime}\right)\right)$

$\exists q \in \operatorname{Hinsicht}^{\prime}\left(\operatorname{dim}^{\prime}\left(\lambda s^{+} . \exists s\left[s \leq s^{+} \wedge \operatorname{attraktiv}^{\prime}\left(\iota l\left[\operatorname{Lösung}^{\prime}(l)\right]\right)(s)\right]\right)\right)$

[in-Hinsicht-auf' $\left(s^{*}, \lambda s^{+} . \exists s\left[s \leq s^{+} \wedge\right.\right.$ attraktiv' $\left(\iota l\left[\right.\right.$ Lösung $\left.\left.\left.\left.^{\prime}(l)\right]\right)(s)\right], q^{f}\right)$, wobei $q^{f}=q$, das über das Fakt $f$ zu erschließen ist] 
Im Anschluss an die oben skizzierte Idee, dass die Dimensionen topiksituationaler Prädikationen Fakten sind, kann in diesem Fall $q$ direkt mit $f$ selbst identifiziert werden; inklusive Bindung der Topiksituation ergeben sich die finalen Wahrheitsbedingungen in (103).

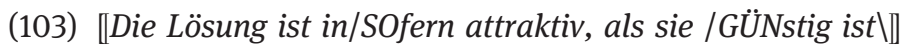

$=1$ gdw. $\exists s^{*} \exists f \in \operatorname{exempl}^{\prime}\left(\lambda s^{\prime}\right.$.günstig' $\left.\left(l l\left[\operatorname{Lösung}^{\prime}(l)\right]\right)\left(s^{\prime}\right)\right)$

[in-Hinsicht-auf ${ }^{\prime}\left(s^{*}, \lambda s^{+} . \exists s\left[s \leq s^{+} \wedge\right.\right.$ attraktiv'(ll[Lösung'(l)])(s)],f)]

Im Ergebnis wird damit wie erwünscht die Sachverhaltsbeschreibung des Matrixsatzes dadurch dimensional spezifiziert, dass sie auf die vom als-Satz beigesteuerte Tatsache relativiert wird: Der Satz (98a) ist wahr gdw. es eine Topiksituation gibt, über die - relativ zur Tatsache, dass die Lösung günstig ist prädiziert wird, dass die Lösung attraktiv ist. Welche Vorteile vereint nun diese Analyse?

Erstens basiert der Vorschlag auf allein unabhängig motivierten Komponenten: dem bereits für LIA-Gefüge genutzten uniformen Lexikoneintrag für insofern als, einer strukturabhängigen Komposition sowie der Annahme, dass dimensionale Spezifizierungen von Sachverhaltsbeschreibungen auf relativierenden Tatsachen beruhen.

Zweitens erfasst die Analyse, warum in SIA-Gefügen keine unmittelbare Sensitivität bezüglich lexikalisch angelegter Mehrdimensionalität besteht. Die Präsupposition einer mehrdimensionalen Prädikation betrifft qua strukturbedingter Komposition die topiksituationale Matrixprädikation, nicht aber deren lexikalische Binnenkomponenten. Entscheidend ist entsprechend, ob die topiksituationale Prädikation mehrdimensional, also über eine sie spezifizierende Tatsache dimensional beschränkbar ist. So ist z. B. das SIA-Gefüge in (104a) (= [51a]) mit eindimensionalem Matrixprädikat schwer deshalb grammatisch, weil der als-Satz eine im Vergleich zur Matrixprädikation spezifischere Tatsache einführt, relativ zu der die Matrixprädikation als wahr behauptbar ist, s. die wohlgeformte Repräsentation in (104b).

(104) a. Ben ist in/SOfern schwer, als er hundert /KIlo wiegt \.

b. 1 gdw. $\exists s^{*} \exists f \in \operatorname{exempl}^{\prime}\left(\lambda s^{\prime} .100-K_{1}\right.$ ilo-wieg'(Ben $\left.)\left(s^{\prime}\right)\right)$

[in-Hinsicht-auf' $\left(s^{*}, \lambda s^{+} . \exists s\left[s \leq s^{+} \wedge \operatorname{schwer}^{\prime}(\right.\right.$ Ben $\left.\left.\left.)(s)\right], f\right)\right]$

Gleichzeitig erklärt sich, warum man hingegen ein Beispiel wie (105) (= [3b]) nicht ohne Weiteres interpretieren kann.

(105) \# Ben ist insofern $1,90 \mathrm{~m}$ groß, als er .... 
Entscheidend ist, dass die topiksituationale Matrixprädikation Ben ist 1,90 m groß eine so spezifische Information beiträgt, dass kaum eine dimensional beschränkende noch spezifischere Tatsache denkbar ist. Das heißt: In diesem Fall wird tatsächlich die von insofern als ausgehende Präsupposition an die topiksituationale Matrixprädikation nicht erfüllt. Bestätigung für diese Perspektive liefert die Beobachtung, dass ungewöhnliche Kontextbedingungen entsprechende SIA-Gefüge dennoch lizenzieren können: Wenn man einen Kontext annimmt, in dem es allein darauf ankommt, dass jemand mindestens 1,90 groß ist (also die für Aussagen wie Ben ist 1,90 gro $\beta$ standardisierte skalare Implikatur Ben ist genau 1,90 gro $\beta$ aushebelt), gibt es Fakten, die spezifischer sind als die gegebene Matrixprädikation. Entsprechend sind SIA-Gefüge hier möglich, s. (106).

(106) [Kontext: Gesucht werden Modells, die mindestens 1,90 groß sind.]

Ben ist insofern 1,90 groß, als er 1,94 groß ist, Paul ist insofern 1,90 groß, als er 1,97 groß ist; Hanno hingegen ist insofern keine 1,90 groß, als er 1,87 groß ist.

Drittens passt der Analysevorschlag dazu, dass insofern als keine Subjektivitätseffekte, d.h. insbesondere auch keine subjektiv-epistemische Einstellung, involviert. So führt insofern als gemäß Eintrag in (87) weder einen subjektiven Urteiler noch eine Einstellung explizit ein; stattdessen geht es allein um die Frage, ob die vom als-Satz eingeführte Tatsache ein plausibler objektiver Kandidat für die dimensionale Spezifizierung der topiksituationalen Matrixprädikation darstellt oder nicht, s. dazu z. B. das wiederholte Beispiel in (107a) (= [66b]) und dessen Wahrheitsbedingungen in (107b).

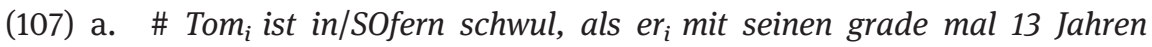
keine /FREUNdin hat $\backslash$.

b. \# 1 gdw. $\exists s^{*} \exists f \in \operatorname{exempl}^{\prime}\left(\lambda s^{\prime}\right.$.mit-13-keine-Freundin-hab'(Tom)(s')) [in-Hinsicht-auf' $\left.\left(s^{*}, \lambda s^{+} . \exists s\left[s \leq s^{+} \wedge \operatorname{schwul}^{\prime}(\mathrm{Tom})(s)\right], f\right)\right]$

(108) Tom $_{i}$ könnte in/SOfern schwul sein, als er ${ }_{i}$ mit 19 Jahren immer noch keine /FREUNdin hat।.

(107b) weist (107a) deshalb als abweichend aus, weil die Tatsache, dass Tom mit 13 Jahren keine Freundin hat, keine Tatsache ist, die die Aussage, dass Tom schwul ist, als solche spezifizieren könnte. Wie mehrfach betont ändert sich die Situation, wenn wie in (108) die Matrixprädikation selbst eine subjektivepistemische Komponente beisteuert: Die Tatsache, dass Tom mit 19 keine 
Freundin hat, kann als spezifische Tatsache aufgefasst werden, relativ zu der als möglich behauptet werden kann, dass Tom schwul ist.

Abschließend möchte ich die Frage diskutieren, ob man das Verhältnis zwischen topiksituationaler Prädikation $p$ und der diese spezifizierenden Tatsache $f$ präziser fassen kann als durch die bisher primär intuitiv bestimmte Vorstellung, dass $f$ ein spezifischeres Bild der von $p$ ausgewählten Topiksituationen liefert. Vorschlagen möchte ich dazu, diese Relativierung als Exemplifikationsrelation $\mathrm{zu}$ verstehen, s. dazu die Definition in (109).

(109) Sei $s^{*}$ eine Topiksituation, $p$ eine topiksituationale Prädikation und $f$ ein Fakt, dann ist in-Hinsicht-auf $\left(s^{*}, p, f\right)$ wahr gdw. $p$ in $s^{*}$ wahr ist und $f p$ exemplifiziert.

Der „Clou“ von SIA-Gefügen besteht damit im Folgenden: Die vom als-Satz bezeichnete Tatsache wird als minimale Situation dafür präsentiert, dass man die Matrixprädikation als wahr behaupten kann. Mit anderen Worten: $p$ wird als wahr de re von $f$ präsentiert. ${ }^{21}$

Die vorgeschlagene Definition bildet direkt ab, dass die vom als-Satz eingeführten Tatsachen als relativ spezifische Entitäten ins Spiel kommen. Ähnlich zum Befund bei LIA-Gefügen wird also auch für SIA-Gefüge eine Asymmetrie zwischen als-Satz und Matrixsatz vorausgesagt. Dass die Matrixprädikation hinreichend unspezifisch sein muss, hat bereits die Diskussion zu den Beispielen (105)/(106) gezeigt. Analoge Evidenz für ein asymmetrisches Verhältnis liefern die Kontraste in (110).

(110) a. Die Lösung ist in/SOfern attraktiv, als sie /GÜNstig ist .

b. \# Die Lösung ist in/SOfern günstig, als sie attrak/TIV ist $\backslash$.

c. Die Lösung ist in/SOfern günstig, als sie (nur)/DREIßig Euro kostet $\backslash$.

d. \# Die Lösung kostet in/SOfern (nur) dreißig Euro, als sie /GÜNstig ist $\backslash$.

Ins Bild passen außerdem zwei weitere Beobachtungen: Man darf mit dem alsSatz keine relativ zur Matrixaussage zu schwache Situation auswählen, stillschweigend annehmend, dass man diese dann so weit spezifizieren kann, bis sich eine plausible Exemplifikation für die Matrixaussage einstellt, s. dazu (111): Wenn Paul 1,90 groß ist, ist wahr, dass er (mindestens) 1,60 groß ist. Dennoch kann die schwächere Situationsbeschreibung, dass er (mindestens) 1,60 groß

21 Diese Art der Formulierung ist Kratzer (2002) entlehnt. Ihr Anliegen besteht darin, die zentrale Rolle von exemplifizierenden Fakten für Wissenszuschreibungen zu motivieren. Im Ergebnis hält sie fest, dass die Aussage $S$ weiß $p$ u. a. bedingt, dass $S p$ de re von $f$ glaubt. 
ist, nicht als Anker für eine solche Erweiterung und damit als dimensionale Spezifizierung der Matrixprädikation, dass Paul groß ist, genutzt werden.

(111) \# Paul ist in/SOfern groß, als er (mindestens) eins/SECHzig ist $\$.

Umgekehrt sollte die spezifizierende Tatsache gemäß Definition (109) auch keine irrelevanten Informationen enthalten. Die folgenden Beispiele bestätigen diese Restriktion:

(112) a. \# Paul ist in/SOfern groß, als er eins/NEUNzig ist und seine Frau /LEberkäse mag\.

b. \# Wir haben in/SOfern Teekannen zur Verfügung, als oben auf dem Dachboden noch 3 /TEEkannen und 12 /GLÄser stehen\.

So wäre in einer Situation s, in der Paul 1,90 ist und seine Frau Leberkäse mag, wahr, dass Paul groß ist. $s$ wäre aber kein Fakt, das die topiksituationale Prädikation, dass Paul groß ist, exemplifizieren würde. Denn die Teilsituation, dass seine Frau Leberkäse mag, wäre nicht zu einer minimalen Situation erweiterbar, für die gilt, dass Paul groß ist. Eine analoge Überlegung trifft auf Beispiel (112b) zu. Die in die Exemplifikationsrelation eingebaute Minimalitätsbedingung macht also die richtige Voraussage für das Verhältnis von als-SatzProposition und Aussage im Matrixsatz.

Ein(e) anonyme(r) Gutachter*in sieht in Beispiel (113a) ein potenzielles Problem für die vorgeschlagene Analyse. Sie/Er argumentiert, dass der Satz in (113a) abweichend ist, aber akzeptabel sein sollte, da doch die vom als-Satz gelieferte Tatsache hier spezifischer ist als die Situationsbeschreibung auf der Matrixebene. Die Kritik zielt dabei nicht auf die anhand der Beispiele in (105)/ (106) diskutierte Ausschaltung einer generalisierten Implikatur, sondern auf den Umgang mit der relativen Unschärfe der Matrixprädikation. Ergänzen möchte ich deshalb Beispiel (113b), für das der Bezug auf den Unschärfebereich der Matrixprädikation noch klarer hervortritt.

(113) a. \# Ben ist insofern 1,90 groß, als er 190,3 cm groß ist.

b. \# Ben ist insofern 1,90 groß, als er $189,7 \mathrm{~cm}$ groß ist.

Meine Erklärung ist Folgende: In Abschnitt 3.2.1 habe ich gezeigt, dass IA-Gefüge nicht dazu dienen, Satisfaktionskriterien für vage Prädikate zu bestimmen. Das Problem an Beispielen wie (113) ist nun, dass der Rückgriff auf die präzisen Zahlen 190,3 cm bzw. 189,7 cm nahelegt, dass es nicht um die damit verknüpften Fakten als solche geht, sondern um den für die Interpretation des sprachlichen 
Ausdrucks 1,90 groß angelegten Präzisierungsgrad. Damit würde aber der alsSatz keine topiksituationale Dimension, sondern Satisfaktionskriterien bestimmen. Aus dieser Warte sind die Beispiele keine Evidenz gegen den vorliegenden Analysevorschlag; sie bestätigen vielmehr, dass der metasprachliche Effekt von IA-Gefügen nicht in der Festlegung von Satisfaktionskriterien liegt, sondern in der Spezifizierung von Dimensionen über sprachunabhängige Fakten; s. dazu auch den folgenden Abschlusskommentar.

Zuletzt sei auf eine Konsequenz der Definition in (109) verwiesen, die zunächst irritieren mag, bei genauerem Hinsehen aber noch klarer hervortreten lässt, was den metasprachlichen Charakter von insofern als im Fall von SIAGefügen ausmacht: Gemäß (109) exemplifiziert die vom als-Satz eingeführte Tatsache $f$ die topiksituationale Matrixprädikation; gemäß logischer Form für SIA-Gefüge wie in (103) gilt aber ja auch, dass $f$ die als-Satz-Proposition exemplifiziert. Dies scheint auf eine widersinnige Symmetrie zwischen Matrixsatz und als-Satz hinauszulaufen. Dieser Eindruck täuscht aber: Die Situationsbeschreibung im als-Satz dient allein dazu, ein relevantes $f \mathrm{zu}$ bestimmen; es wird nichts $\mathrm{zu}$ den dafür gewählten Worten gesagt, sondern stillschweigend angenommen, dass man mit z. B. (als) die Lösung günstig ist oder (als) Ben 1,90 groß ist auf das entsprechende Fakt in der Welt tatsächlich referieren kann. Für die topiksituationale Matrixprädikation ist dies grundlegend anders. Das sprachunabhängige Fakt $f$ wird auf dieser Ebene via Exemplifikationsrelation explizit als relevanter Anker für die sprachabhängige Interpretation dieser topiksituationalen Matrixprädikation ausgewiesen. Mit anderen Worten: Die Exemplifikationsrelation dient hier nicht dazu, ein Fakt herauszugreifen, sondern dazu, etwas über die auf der Matrixebene gewählten Worte zu sagen, nämlich zu sagen, dass man diese so interpretieren muss, dass $f$ als Exemplifikation für sie verstanden werden kann. In genau diesem Sinne wird mit der topiksituationalen Matrixprädikation kein neues Fakt eingeführt, sondern eine Interpretation ausgewählt.

\section{Fazit}

Der vorliegende Beitrag hatte die kompositionale Interpretation von insofern als-Gefügen (= IA-Gefüge) zum Gegenstand. Auf der Basis struktursensitiver Tests (Skopus von Negation und Fokuspartikeln, Binnenprojektion in lexikalischen Phrasenkategorien, Fokus-Hintergrund-Gliederung, Bindungsverhalten) habe ich für die Unterscheidung zwischen integrierten IA-Gefügen mit lexikalischem Bezug (= LIA-Gefügen) und sententialen desintegrierten Pendants (= SIA-Gefügen) argumentiert. Der uniforme Bedeutungsbeitrag von IA-Gefügen 
besteht in der dimensionalen Spezifizierung einer präsupponierten mehrdimensionalen Bezugsgröße. Gleichzeitig sorgt der Strukturunterschied in regulärer Weise für eine jeweils verschiedene Identifikation dieser Bezugsgröße: LIA-Gefüge dienen der dimensionalen Einschränkung einer mehrdimensionalen lexikalischen Prädikation des Matrixsatzes, während SIA-Gefüge die durch den Matrixsatz gelieferte Sachverhaltsbeschreibung als Ganze auf eine Dimension relativieren. Zur Stützung dieser Analyseperspektive aus semantisch-pragmatischer Perspektive habe ich folgende Evidenzen angeführt: LIA-, nicht aber SIAGefüge sind sensitiv bezüglich im Matrixsatz lexikalisch angelegter Mehrdimensionalität. Demgegenüber ist für SIA-Gefüge entscheidend, ob der als-Satz eine spezifizierende Information liefert, relativ zu der die vom Matrixsatz gegebene Sachverhaltsbeschreibung als objektiv gültig behauptet werden kann; dies erklärt vor allem, warum SIA-Gefüge Skopus über Modale und Einstellungsbeschreibungen haben können. Dass der wesentliche metasprachliche Effekt von IA-Gefügen in ihrer dimensionsbeschränkenden Funktion liegt, ließ sich außerdem durch negative Ergebnisse für alternative Deutungen stützen: IA-Gefüge dienen nicht dazu, Matrixprädikationen zu disambiguieren oder ihnen Satisfaktionskriterien aufzulegen. Insbesondere fehlen - im Kontrast zum Einstellungsverb finden - Anzeichen dafür, dass IA-Gefüge eine spezifisch subjektive Bedeutung involvieren. So gibt es keine Selektionsbeschränkung auf urteilersensitive Prädikationen; weder forcieren sie die Einführung einer sprachlich zugänglichen subjektiven Einstellung noch befördern sie die Immunisierung gegen Widerspruch.

Den Abschluss der Untersuchung bildete eine Formalisierungsskizze der Komposition. Demnach lizenziert die strukturelle Integration von LIA-Gefügen die dimensionale Beschränkung einer lexikalischen Binnenprädikation des Matrixsatzes; korrespondierend liefert eine aus dem als-Satz konzeptuell zu erschließende lexikalisch-deskriptive Hinsicht den relevanten Dimensionsparameter. Anders SIA-Gefüge: Diese lizenzieren dank Desintegration den holistischen Zugriff auf die topiksituationale Matrixprädikation. Ich habe dafür plädiert, deren dimensionale Spezifizierung als eine Relativierung auf Tatsachen $\mathrm{zu}$ verstehen. Entsprechend liefert der als-Satz eine Tatsache, die ein relativ spezifischeres Bild der gegebenen Situationsbeschreibung auf der Matrixebene liefert und damit de re die Behauptbarkeit der topiksituationalen Matrixprädikation rechtfertigt.

Danksagung: Der vorliegende Beitrag wurde von Projekt A1 des DFG-geförderten Sonderforschungsbereichs 833 „Bedeutungskonstitution“ unterstützt. Für wertvolle Kommentare und Verbesserungsvorschläge danke ich sehr herzlich zwei anonymen Gutachtern sowie Katrin Axel-Tober, Sigrid Beck, Veronika Ehrich, Hans Kamp, Claudia Maienborn, Marga Reis und Carla Umbach. Dank 
schulde ich darüber hinaus den Zuhörer*innen im Semantikzirkel am LeibnizZentrum Allgemeine Sprachwissenschaft in Berlin, wo ich die hier ausgearbeiteten Ideen erstmals vorstellen und diskutieren durfte.

\section{Literatur}

Antomo, Mailin \& Markus Steinbach. 2010. Weil-V2-Sätze an der Schnittstelle zwischen Syntax, Semantik und Pragmatik. Zeitschrift für Sprachwissenschaft 29(1). 1-38.

Barker, Chris. 2002. The dynamics of vagueness. Linguistics and Philosophy 25(1). 1-36.

Bartsch, Renate. 1986. The construction of properties under perspectives. Journal of Semantics 5(4). 293-320.

Beck, Sigrid. 2011. Comparison constructions. In Klaus von Heusinger, Claudia Maienborn \& Paul Portner (eds.), Semantics: An international handbook of natural language meaning. Volume 2, 1341-1390. Berlin \& Boston: de Gruyter.

Breindl, Eva, Anna Volodina \& Ulrich Hermann Waßner. 2014. Handbuch der deutschen Konnektoren 2. Semantik der deutschen Satzverknüpfer. Berlin \& Boston: de Gruyter.

Bücking, Sebastian. 2012. Müdigkeit und Müde-Sein: Zur Semantik adjektivbasierter Zustandsnominalisierungen im Deutschen. Linguistische Berichte 232. 361-397.

Bücking, Sebastian. 2015. Zur Syntax hypothetischer Vergleichssätze im Deutschen. Zeitschrift für germanistische Linguistik 43(2). 261-305.

Bücking, Sebastian. 2017. Composing wie wenn - the semantics of hypothetical comparison clauses in German. Natural Language and Linguistic Theory 35(4). 979-1025.

Christ, Rüdiger. 2014. Zur Satzfügung im Deutschen - vornehmlich am Beispiel adversativer Satzgefüge. Tübingen: Stauffenburg.

Demske, Ulrike. 2014. Verbstellungsvariation in hypothetischen Vergleichssätzen. Linguistische Berichte 238. 101-140.

Duden. 2015. Duden - Das Aussprachewörterbuch (Duden Bd. 6). Bearb. von Stefan Kleiner und Ralph Knöbel in Zusammenarbeit mit der Dudenredaktion. 7., komplett überarb. u. aktual. Aufl. Berlin: Dudenverlag.

Duden. 2016. Duden - Die Grammatik (Duden Bd. 4). Hrsg. von Angelika Wöllstein und der Duden-Redaktion. 9., vollständig überarb. u. aktual. Aufl. Berlin: Dudenverlag.

Frey, Werner. 2006. Contrast and movement to the German prefield. In Valéria Molnár \& Susanne Winkler (eds.), The architecture of focus, 235-264. Berlin \& Boston: de Gruyter.

Frey, Werner. 2011. Peripheral adverbial clauses, their licensing, and the prefield in German. In Eva Breindl, Gisella Ferraresi \& Anna Volodina (eds.), Satzverknüpfung - Zur Interaktion von Form, Bedeutung und Diskursfunktion, 41-77. Berlin \& Boston: de Gruyter.

Hobbs, Jerry J., Mark E. Stickel, Douglas E. Appelt \& Paul Martin. 1993. Interpretation as abduction. Artificial Intelligence 63. 69-142.

Kennedy, Christopher. 1999. Projecting the adjective: The syntax and semantics of gradability and comparison. New York: Garland.

Kennedy, Christopher. 2013. Two sources of subjectivity: Qualitative assessment and dimensional uncertainty. Inquiry 56(2-3). 258-277.

Klein, Wolfgang. 1994. Time in language. London: Routledge.

Kratzer, Angelika. 2002. Facts: Particulars or information units? Linguistics and Philosophy 25(5-6). 655-670. 
Kratzer, Angelika. 2010. Situations in natural language semantics. In Edward N. Zalta (ed.), The Stanford Encyclopedia of Philosophy (Fall 2010 Edition), https://plato.stanford. edu/ archives/fall2010/entries/situations-semantics/ (31.01.2018).

Krifka, Manfred. 2013. Definitional generics. In Alda Mari, Claire Beyssade \& Fabio del Prete (eds.), Genericity, 372-389. Oxford: Oxford University Press.

Krifka, Manfred \& Renate Musan. 2012. Information structure: Overview and linguistic issues. In Manfred Krifka \& Renate Musan (eds.), The expression of information structure, 1-44. Berlin \& Boston: de Gruyter.

Maienborn, Claudia. 2001. On the position and interpretation of locative modifiers. Natural Language Semantics 9(2). 191-240.

Maienborn, Claudia. 2003a. Die logische Form von Kopula-Sätzen. Berlin: Akademie Verlag.

Maienborn, Claudia. 2003b. Event-internal modifiers: Semantic underspecification and conceptual interpretation. In Ewald Lang, Claudia Maienborn \& Cathrine FabriciusHansen (eds.), Modifying adjuncts, 475-509. Berlin \& Boston: de Gruyter.

Maienborn, Claudia. 2005. On the limits of the Davidsonian approach: The case of copula sentences. Theoretical Linguistics 31(3). 275-316.

Moltmann, Friederike. 2009. Degree structure as trope structure: A trope-based analysis of positive and comparative adjectives. Linguistics and Philosophy 32(1). 51-94.

Moltmann, Friederike. 2013. Abstract objects and the semantics of natural language. Oxford: Oxford University Press.

Reich, Ingo \& Marga Reis. 2013. Koordination und Subordination. In Jörg Meibauer, Markus Steinbach \& Hans Altmann (eds.), Handbuch der Satztypen, 535-568. Berlin \& Boston: de Gruyter.

Reis, Marga. 1997. Zum syntaktischen Status unselbständiger Verbzweit-Sätze. In Christa Dürscheid, Karl-Heinz Ramers \& Monika Schwarz (eds.), Syntax im Fokus. Festschrift für Heinz Vater, 121-144. Tübingen: Niemeyer.

Reis, Marga. 2011. Kaum-Gefüge im Deutschen - Grammatik und Pragmatik. Zeitschrift für germanistische Linguistik 39(3). 317-355.

Reis, Marga. 2013a. Dt. finden und „subjektive Bedeutung“. Linguistische Berichte 236. 389-426.

Reis, Marga. 2013b. „Weil-V2“-Sätze und (k)ein Ende? Anmerkungen zur Analyse von Antomo \& Steinbach (2010). Zeitschrift für Sprachwissenschaft 32(2). 221-262.

Reis, Marga \& Angelika Wöllstein. 2010. Zur Grammatik (vor allem) konditionaler V1-Gefüge im Deutschen. Zeitschrift für Sprachwissenschaft 29(1). 111-180.

Sæbø, Kjell Johan. 2009. Judgment ascriptions. Linguistics \& Philosophy 32(4). 327-352.

Sassoon, Galit W. 2013a. A typology of multidimensional adjectives. Journal of Semantics 30(3). 335-380.

Sassoon, Galit W. 2013b. Vagueness, gradability and typicality. The interpretation of adjectives and nouns. Leiden: Brill.

Speyer, Augustin. 2011. Je stärker der Fokus, desto geringer die Einbettung? Zum Status des je-Satzes in je-desto-Gefügen. Linguistische Berichte 225. 43-61.

Umbach, Carla. 2016. Evaluative propositions and subjective judgments. In Cécile Meier \& Janneke Wijnbergen-Huitink (eds.), Subjective meaning. Alternatives to relativism, 127-168. Berlin \& Boston: de Gruyter.

Umbach, Carla \& Helmar Gust. 2014. Similarity demonstratives. Lingua 149. 74-93.

Zifonun, Gisela, Ludger Hoffmann \& Bruno Strecker. 1997. Grammatik der deutschen

Sprache. 3 Bde. Berlin \& Boston: de Gruyter. 